\title{
1 Insights into natural products biosynthesis from analysis of 490 polyketide synthases from Fusarium
}

3

4

Daren W. Brown* and Robert H. Proctor

Mycotoxin Prevention and Applied Microbiology

U.S. Department of Agriculture, Agricultural Research Service, National Center for Agricultural Utilization Research, 1815 N. University St., Peoria, Illinois 61604, *Corresponding author. Tel: (309)-681-6230; Fax: (309)-681-6672; e-mail: daren.brown@ars.usda.gov

\section{Abstract}

Species of the fungus Fusarium collectively cause disease on almost all crop plants and produce numerous natural products (NPs), including some of the mycotoxins of greatest concern to agriculture. Many Fusarium NPs are derived from polyketide synthases (PKSs), large multidomain enzymes that catalyze sequential condensation of simple carboxylic acids to form polyketides. To gain insight into the biosynthesis of polyketide-derived NPs in Fusarium, we retrieved 488 PKS gene sequences from genome sequences of 31 species of the fungus. In addition to these apparently functional PKS genes, the genomes collectively included 83 pseudogenized PKS genes. Phylogenetic analysis resolved the PKS genes into 67 clades, and based on multiple lines of evidence, we propose that homologs in each clade are responsible for synthesis of a polyketide that is distinct from those synthesized by PKSs in other clades. The presence and absence of PKS genes among the species examined indicated marked differences in distribution of PKS homologs. Comparisons of Fusarium PKS genes and genes flanking them to those from other Ascomycetes provided evidence that Fusarium has the genetic potential to synthesize multiple NPs that are the same or similar to those reported in other fungi, but that have not yet been reported in Fusarium. The results also highlight ways in which such analyses can help guide identification of novel Fusarium NPs and differences in NP biosynthetic capabilities that exist among fungi.

Keywords: Fusarium; secondary metabolite; natural product; polyketide; evolution; polyketide synthase 


\section{Introduction}

Fungi produce thousands of structurally diverse metabolites that are not essential for growth or reproduction (Cole et al., 2003). Such compounds are referred to as secondary metabolites or natural products (NPs), and collectively they have a broad range of biological activities (Keller, 2015; Keller et al., 2005). Some have beneficial pharmaceutical activities, some are toxic to animals and/or plants, others are plant hormones, and still others are pigments. Although the roles of most NPs in the ecology of fungi that produce them are not known, some contribute to virulence on plants or insects, while others enhance competitiveness with other microbes. Over the past two decades, there has been a tremendous effort to understand the genetic and biochemical pathways that lead to the formation of NPs, because of the potential for such information to contribute to drug discovery, efforts to control plant disease and toxin contamination problems caused by fungi, as well as development of effective biological control strategies for insect and microbial pests (Ahuja et al., 2012; Brown et al., 2014).

The majority of fungal NPs characterized to date are derived from polyketides (Brown et al., 2015; Wiemann and Keller, 2014; Wiemann et al., 2013). Fungal polyketide synthases (PKSs) are large, multi-domain proteins that catalyze sequential (or iterative) condensation of simple carboxylic acids, such as malonate (Hopwood and Khosla, 1992). The broad structural diversity of polyketides arises from: different carboxylic acids substrates used by PKSs; whether the $\beta$-carbonyl formed during each condensation cycle is reduced; and whether the polyketide is cyclized. The even greater structural diversity of polyketide-derived NPs results from post-PKS structural modifications, such as oxygenation, acylation, and further cyclization. In fungi, the genes encoding a PKS and polyketide-modifying enzymes required for synthesis of the same NP, are typically located next to one another in gene clusters (Brown et al., 1996). Such clusters also typically include a gene encoding a protein that transports the $\mathrm{NP}(\mathrm{s})$ across the cell membrane, as well as a gene encoding a transcription factor that regulates expression of the cluster genes.

Genomic studies indicate that fungi have the genetic potential to produce many more NPs than predicted based on chemical analyses of fermentation extracts (Cuomo et al., 2007; Ehrlich and Mack, 2014; Gaffoor et al., 2005; Georgianna et al., 2010). Significant effort has been invested to identify the polyketides and/or polyketide-derived NPs formed via the activities of PKSs identified through fungal genome sequencing. These efforts require a combination of molecular genetics and analytical chemistry approaches and, as a result, can be demanding of 
time and resources. Fungal NP biosynthetic gene clusters are often not expressed under laboratory culture conditions. Thus, one approach to identify a polyketide product of a PKS is to overexpress the corresponding PKS gene and/or the transcription factor gene located within the same NP gene cluster (Ahuja et al., 2012; Connolly et al., 2013; Jorgensen et al., 2014). Although such efforts have achieved significant success, the polyketides synthesized by many fungal PKSs identified by genome sequencing have yet to be identified. For example, in Aspergillus nidulans the polyketide products of 11 of 27 PKSs and in Fusarium graminearum the products of seven of 15 PKSs have not been identified (Ahuja et al., 2012; Gaffoor et al., 2005; Hansen et al., 2015; Jorgensen et al., 2014; Sorensen et al., 2014).

Phylogenetic and other comparative analyses of PKS homologs from multiple fungi can guide efforts to identify novel NPs and gain insights into NP biosynthetic pathways (Throckmorton et al., 2015). Different genera of fungi as well as different species within the same genus can produce the same or structurally similar NPs and therefore have relatively closely related homologs of NP biosynthetic genes, including PKS genes. For example, some species of the relatively distantly related fungi Fusarium (Sordariomycetes) and Aspergillus (Eurotiomycetes) produce fumonisins, a group of structurally related polyketide-derived mycotoxins (Susca et al., 2014). Thus, it is often possible to gain significant insight into the genetic potential of a fungus to produce NPs by comparing its putative NP biosynthetic genes to those from other fungi in which the genetics of NP biosynthesis has been more thoroughly characterized and genetic tools are readily available.

Fusarium includes over 100 described species that collectively cause disease on an extraordinarily wide range of plant species, including most crops (Leslie and Summerell, 2006; Morgavi and Riley, 2007). Fusarium is also reported to produce over 50 structurally related families of NPs that vary tremendously in chemical structure and biological activity (Desjardins, 2006; Vesonder and P., 1989). Among these NPs are mycotoxins (e.g. fumonisins, trichothecenes and zearalenone) that can contaminate food and feed derived from Fusariuminfected crops. The economic impact of mycotoxin contamination via its effects on health of humans and livestock as well as on international trade is estimated to be in the 100s of millions of US dollars each year (Wu, 2007).

Comparative analyses of Fusarium PKS genes and associated gene clusters can provide important insights into the genetic potential of species of the fungus to produce NPs as well as 
guide efforts to identify the corresponding polyketides and NPs (Brown et al., 2012a; Brown and Proctor, 2013; Hansen et al., 2015; Hansen et al., 2012; Ma et al., 2010; Wiemann et al., 2013). Such analyses can also provide insights into the distribution and evolution of NP biosynthetic genes. Most recently, 153 PKSs have been cataloged from analyses of genome sequences of 10 Fusarium species: F. acuminatum, $F$. circinatum, F. culmorum, $F$. fujikuroi, $F$. graminearum, $F$. mangiferae, F. oxysporum, F. pseudograminearum, F. solani f. sp. pisi, and F. verticillioides (Hansen et al., 2015). Phylogenetic analyses have resolved the PKSs into two major clades, or groups: a non-reducing PKS (NR-PKS) group responsible for synthesis of aromatic polyketides; and a reducing PKS (R-PKS) group responsible for synthesis of mostly nonaromatic polyketides (Brown et al., 2012a; Ma et al., 2010; Wiemann et al., 2013). In addition, the R-PKS group has been further resolved into three subclades, designated R-PKS I, II and III. The resolution of Fusarium PKSs into the NR-PKS and three R-PKS groups is consistent with results of similar analyses of PKSs from multiple fungal genera (Baker et al., 2006; Kroken et al., 2003; Riley et al., 2014). Hansen et al. (2015) classified the 153 PKSs from Fusarium into 52 orthology groups and summarized information on the polyketide-derived NP products formed via the activities of orthologs in 15 of the groups. Therefore, the NPs corresponding to the other 37 orthology groups have not been identified.

The objective of this study was to gain further insight into the genetics of NP biosynthesis in Fusarium through comparative analysis of PKS genes retrieved from genome sequences of 31 species of the fungus. The results of this analysis provide evidence that the species examined have the collective genetic potential to produce 64 structurally distinct families of polyketide-derived NPs. The results also reveal extensive variability in patterns of distribution of PKS genes among the species and provide evidence for production of seven families of NPs that have not been reported previously in Fusarium. Insights gained from these results should help guide future studies aimed at identifying novel polyketides and polyketidederived NPs produced by Fusarium and other fungi.

\section{Materials and Methods.}

Fusarium species and DNA sequence data generation. The Fusarium species examined in this study are listed in Table 1. Genome sequence data for 11 Fusarium species were obtained from the National Center for Biotechnology Information (NCBI) (www.ncbi.nlm.nih.gov). 
Whole-genome sequencing was performed on DNA extracted from 20 Fusarium species grown in GYP (2\% glucose, $1 \%$ peptone, and $0.3 \%$ yeast extract) liquid cultures using the ZR Fungal DNA MiniPrep ${ }^{\mathrm{TM}}$ kit as described by the manufacturer (Zymo Research, Irvine CA). Genome sequence data for seven Fusarium species were generated by the Beijing Genome Institute, Hong Kong) using a HiSeq 2000 sequencing platform. Genome sequence data for 13 Fusarium species were generated using a Life Technologies Ion Torrent PGM ${ }^{\mathrm{TM}}$ (12 genomes) or Illumina MiSeq ${ }^{\circledR}$ (one genome) sequencing platform at NCAUR-ARS. The genomic DNA libraries for sequencing with Ion Torrent were constructed from $1 \mu \mathrm{g}$ of purified DNA using the New England Biolabs NEBnext Fast DNA Library prep set for Ion Torrent. The sequencing data was obtained using the Ion Torrent $400 \mathrm{bp}$ template kit and the HiQ sequencing chemistry on a 318v2 chip. The genomic DNA library for sequencing with the Illumina MiSeq was constructed from 1 ng of purified DNA using the Nextera XT DNA Library Preparation Kit. The sequencing data was obtained using the MiSeq Reagent Kit v3. Low-quality reads were trimmed and filtered using CLC Genomics Workbench (version 8.0) and assembled into contigs.

Search for PKSs and housekeeping genes. Putative functional and non-functional PKSs (e.g. pseudogenes) and housekeeping genes were identified by Basic Local Alignment Search Tool (BLAST) analysis (Zhang et al., 1998) against BLAST databases for all 31 Fusarium genome sequence data sets created in CLC Genomics Workbench. The PKS BLAST query sequences were nucleotide sequences encoding $16 F$. fujikuroi $\mathrm{PKSs}$, three $F$. verticillioides $\mathrm{PKSs}, 10 F$. graminearum $\mathrm{PKSs}$, one $F$. circinatum $\mathrm{PKS}$, four $F$. oxysporum $\mathrm{PKSs}$ and four $F$. solani $\mathrm{f}$. sp. pisi PKSs, which collectively represent the 37 different groups or clades of Fusarium PKSs identified previously by phylogenetic analysis (Brown and Proctor, 2013; Wiemann et al., 2013). Nucleotide sequences with $\mathrm{E}$ values $<1 \times 10^{-10}$ to the query sequence and flanking sequences were subjected to BLASTX analysis against NCBI-NR databases to identify the full-length PKSs and PKS pseudogenes (Supplemental Table 2). Sequence adjacent to select putative PKSs were also subjected to BLASTX analysis against NCBI-NR databases to identify potential NP biosynthetic genes. The housekeeping gene BLAST query sequences were 20 putative housekeeping genes from F. graminearum (Supplemental Table 3 and 4). Nucleotide sequences with $\mathrm{E}$ values $<1 \times 10^{-100}$ to the query sequence and flanking sequences were subjected to BLASTX analysis against NCBI-NR databases to identify each full-length housekeeping gene. 
157 Phylogenetic analysis. The evolutionary history of 490 Fusarium PKSs and 55 PKSs from other Ascomycetes (Supplemental Table 1 and 2) within and among species was inferred using the Maximum Likelihood (ML) method, based on the Tamura-Nei Model (Tamura and Nei, 1993) and the Maximum Parsimony (MP) method in MEGA6 (Tamura et al., 2013). Amino acid sequences corresponding to the 546 predicted proteins were aligned using Muscle and converted to nucleotide sequence (Supplemental Text) prior to constructing the phylogeny. All positions containing gaps and missing data were eliminated. The ML tree with the highest log likelihood is shown (Figure 1) and is rooted on the fatty acid synthase (FAS) from Gallus gallus. The percentage of trees in which the associated taxa clustered together in the bootstrap test (500 replicates) is shown next to the branches. Initial tree(s) for the heuristic search were obtained automatically by applying Neighbor-Join and BioNJ algorithms to a matrix of pairwise distances estimated using the Maximum Composite Likelihood (MCL) approach, and then selecting the topology with the superior log likelihood value. The tree is drawn to scale, with branch lengths measured in the number of substitutions per site. Phylogenetic trees based on the coding sequences for the combined keto-synthase and acyltransferase/malonlytransferase (KSAT/MAT) domains were very similar to the tree based on the entire PKS gene coding region (data not shown). A species phylogeny for the 31 Fusarium species was inferred from 20 housekeeping genes (Supplemental Table 3 and 4) using the same methods used for phylogenetic analysis of the PKS genes except that alignments were generated from nucleotide with ClustalW as implemented in MEGA6 (Figure 2).

\section{Results and Discussion}

Phylogenetic framework of species. To establish a phylogenetic framework of the 31

Fusarium species examined in this study, we retrieved the nucleotide sequences of coding regions of 20 housekeeping genes from genome databases of each species, aligned homologous sequences, and then subjected concatenated alignments of all 20 genes to maximum likelihood and maximum parsimony analysis. The resulting species phylogenies (Figure 2) were largely consistent with previously described phylogenies that included similar sets of species (Geiser et al., 2013; O'Donnell et al., 2013). The phylogeny inferred in the current study indicated that the species examined are distributed among 10 previously described species complexes (Figure 2) (Geiser et al., 2013; O'Donnell et al., 2013). Also consistent with the previous studies, Fusarium 
188

189

190

191

192

193

194

195

196

197

198

199

200

201

202

203

204

205

206

207

208

209

210

211

212

213

214

215

216

217

218

sp. NRRL 25184 was not resolved within a species complex, but instead was resolved as a single-member lineage that is sister to the $F$. fujikuroi, $F$. nisikadoi, and $F$. oxysporum species complexes (Figure 2).

Resolution of Fusarium PKSs into 67 Clades. In this study, we examined 488 PKS gene sequences retrieved from 11 publically available Fusarium genome sequences (171 PKSs) and 20 newly generated Fusarium genome sequences (317 PKSs) (Supplemental Table 2). Our analysis also included two PKS gene sequences from Fusarium heterosporum (for a total of 490 Fusarium PKSs) and 55 PKS sequences (Supplemental Table 1) from other genera of the Ascomycota. The latter PKSs have been functionally characterized and the corresponding polyketides or polyketide-derived NPs have been identified.

The genealogies generated by maximum likelihood and maximum parsimony analysis of the nucleotide sequences of the full-length PKS coding regions (Figure 1) or the KS-AT/MAT domains (data not shown) were similar in topology as well as support for clades. Consistent with previous studies (Brown et al., 2012a; Ma et al., 2010; Wiemann et al., 2013), the current analysis resolved two well supported clades (100\%) corresponding to the NR-PKS and R-PKS II groups of PKSs and a moderately supported clade (79\%) corresponding to R-PKS III (Figure 1). In contrast to the previous studies, however, the current analysis did not resolve R-PKS I PKSs into a well supported clade, but instead resolved them into 21 smaller clades, each with $>99 \%$ bootstrap support, and two single-member PKS clades. As a result, in the current study we assigned PKSs to the R-PKS I groups based on phylogenetic relationships to PKSs previously assigned to this group (Brown et al., 2012a; Wiemann et al., 2013). In previous studies, two PKSs, FCIRG_08230 from F. circinatum and FO47SC12 from F. oxysporum were present on separate branches sister to the NR-PKS clade (Brown et al., 2012a; Wiemann et al., 2013). In this study, a PKS from $F$. redolens and another from $F$. foetens were resolved within the same clade as FO47SC12. These four PKSs are distinct from all other R-PKSs in that they lack either a functional or pseudogenized methyltransferase (ME) domain and, therefore, will be referred to hereafter as Rm-PKSs.

Consistent with previous results, the maximum likelihood analysis in the current study resolved PKSs within the NR-PKS and R-PKS groupings into smaller and well supported clades, which we have designated Clades $1-33,35-40,42-45$ and $48-71$. A subset of these clades correspond, for the most part, to the PKS orthology groups PKS1 - PKS33, PKS35 - PKS40, 
PKS42 - PKS45 and PKS48 - PKS52 recently described by Hansen et al. (2015). For example, Clades $1-33$ correspond to orthology groups $P K S 1-P K S 33$. However, there are a few discrepancies between the clades resolved in the current analysis and the orthology groups described by Hansen et al. (2015). In addition, we also gave clade numbers to 15 Fusarium PKSs that were not resolved into clades with other PKSs: Clades 31, 49, 54 - 60, 62, 64, 67, 68, 70 and 71. PKS Clades $1-33,35-40,42-45$ and $48-71$ were distributed across the larger NR-PKS and three R-PKS groups: the NR-PKS group was resolved into 11 smaller clades, and R-PKS groups I, II and III were resolved into 23, 14 and 17 smaller clades respectively. In addition, the four PKSs in the Rm-PKS group were resolved into two smaller clades. Even though the PKS genes used in our phylogenetic analysis included highly divergent sequences, the phylogenetic analysis with this large and divergent data set was effective at resolving a large number of clades. Phylogenetic analyses with smaller sets of more closely related sequences confirmed relationships resolved with the large data set, but provided a higher resolution for relationships between homologs within clades and, in some cases, between clades (Supplementary Figure 2).

Based on the PKS domain structures and knowledge of the polyketide products synthesized by some of the PKSs, we hypothesize that PKS homologs in each clade are responsible for synthesis of a polyketide that is structurally distinct from polyketides synthesized by PKSs in other clades. If this hypothesis is correct, the 490 Fusarium PKSs analyzed here are likely responsible for synthesis of 67 structurally distinct polyketides; i.e., one polyketide for each of the 67 PKS Clades. The hypothesis is consistent with the location of the PKS genes from the same clade within homologous gene clusters. For example, the five PKSs in Clade 24 are orthologs of the enzyme (Fum1), which is required for biosynthesis of fumonisin mycotoxins (Proctor et al., 1999). In all five species, the FUM1 gene is located in the 16-17 gene fumonisin biosynthetic gene (FUM) cluster (Proctor et al., 2013).

It is likely though that fewer than 67 polyketide-derived NPs, or families of NPs, are formed from the 67 putative polyketides. This is because some NPs are formed via the activity of pairs of PKSs consisting of an R-PKS and an NR-PKS where the R-PKS synthesizes a polyketide that serves as a starter unit for the NR-PKS to synthesize a larger polyketide. Such RPKS/NR-PKS pairs are required for synthesis of zearalenone in Fusarium (Clade 13 and Clade 4) and several other metabolites in Aspergillus (Ahuja et al., 2012; Gaffoor and Trail, 2006; Kim 
250

251

252

253

254

255

256

257

258

259

260

261

262

263

264

265

266

267

268

269

270

271

272

273

274

275

276

277

278

279

280

et al., 2005). The genes encoding these R-PKS/NR-PKS pairs are typically located adjacent or close to one another on a chromosome. In F. fujikuroi, an R-PKS/NR-PKS pair of unknown function located $15 \mathrm{~kb}$ apart was found and proposed to be involved in the synthesis of a single polyketide (Wiemann et al., 2013). The 488 PKS genes that we retrieved from Fusarium genome sequences included four pairs of R-PKS/NR-PKS genes located adjacent to on another. The zearalenone pair, Clade 13 and Clade 4 is present in four Fusarium and the unknown metabolite putative pair from F. fujikuroi, Clade 36 and Clade 37, is present in 10 Fusarium. Two new R-PKS/NR-PKS pair include the R-PKS genes of Clade 48 and NR-PKS genes of Clade 53 in three Fusarium and the R-PKS gene of Clade 62 and the NR-PKS gene of Clade 54 in $F$. thapsinum. Based on their locations next to one another, these additional pairs of RPKS/NR-PKS genes are likely required for synthesis of the same NP. Thus, although we propose that each of the 67 clades of Fusarium PKSs is responsible for synthesis of a structurally distinct polyketide, it is likely that 67 polyketides give rise to only 63 families of NPs.

Discrepancies between Clades and Orthology Groups. Comparison of the orthology groups described by Hansen et al. (2015) with clades resolved by our phylogenetic analysis indicated that most of the orthology groups correspond to well-supported clades. There were, however, four exceptions. The first exception involves orthology groups $P K S 16, P K S 46$ and $P K S 47$ described by Hansen et al. (2015). PKS46 and PKS47 groups each consisted of two PKSs, one from $F$. acuminatum and another from $F$. avenaceum. Our phylogenetic analysis indicated that these PKSs are closely related to PKSs in orthology group PKS16, which consists of PKSs required for synthesis of the pigment bikaverin (Figure 3A). Analysis of genes flanking PKS46 and PKS47 in F. acuminatum and F. avenaceum revealed the presence of homologs of some or all of the genes in the bikaverin biosynthetic gene (BIK) cluster (Figure 3A) (Wiemann et al., 2009). These findings suggest that $F$. acuminatum and $F$. avenaceum have two BIK cluster-like clusters. Given that genes $B I K 1, B I K 2$ and $B I K 3$ are the only enzyme-encoding genes required for bikaverin synthesis (Wiemann et al., 2009), and that these three genes are present in the BIK cluster-like clusters in $F$. acuminatum and $F$. avenaceum, we propose that PKSs in orthology groups PKS46 and PKS47 are likely functional orthologs of the bikaverin PKS and, therefore, we include them in the same grouping Clade 16. These findings also indicate that $F$. acuminatum and $F$. avenaceum have the genetic potential to produce bikaverin and/or a bikaverin-like 
281

282

283

284

285

286

287

288

289

290

291

292

293

294

295

296

297

298

299

300

301

302

303

304

305

306

307

308

309

310

311

metabolite(s). Because PKS46 and PKS47 described by Hansen et al (2015) were included in Clade 16 in the current study, and in an attempt to avoid confusion, we have not included a Clade 46 or 47 in our numbering system. In a previous study, the bikaverin PKS gene (BIK1) was not detected in multiple strains of $F$. avenaceum and $F$ acuminatum by Southern blot analysis using a hybridization probe that consisted of an approximately $1-\mathrm{kb}$ fragment that spanned the ketosynthase (KS)-coding domain of the F. verticillioides BIK1 gene (O'Donnell et al., 2013). The failure to detect BIK1 homologs in $F$. acuminatum and $F$. avenaceum is likely a result of the relatively low level of amino acid sequence identity $(61-67 \%)$ of the $F$. verticillioides BIKI probe (KS domain) to the KS domain of the homologs in the other two species. Previously, based in part on phylogenetic analysis, it was proposed that the bikl from Botrytis cinerea was acquired from Fusarium through horizontal gene transfer (HGT) (Campbell et al., 2012;

Schumacher et al., 2013). Our results are consistent with this hypothesis and further suggest that the donor was likely a species that arose after the $F$. fujikuroi, F. nisikadoi and F. oxysporum species complexes diverged from F. redolens (Supplemental Figure 3).

The second exception involved orthology group PKS2O of Hansen et al. (2015), which consisted of a PKS from $F$. avenaceum, $F$. fujikuroi, $F$. oxysporum and $F$. verticillioides. In our phylogenetic analysis, the $F$. fujikuroi, $F$. oxysporum and $F$. verticillioides $\mathrm{PKSs}$ were resolved as part of a well-supported clade (Clade 20; bootstrap value 100) that did not include the $F$. avenaceum PKS (FAVG1_07658). In fact, FAVG1_07658 resolved as part of a clade (bootstrap value 97) that included the $F$. heterosporum PKS that is responsible for synthesis of the NP fusaridione (Figure 1). Analysis of the genes flanking the Clade 20, Clade 69 and FAVG1_07658 PKS genes revealed that two of the FAVG1_07658 flanking genes were closely related homologs of genes in the proposed five-gene fusaridione biosynthetic gene cluster (Figure 3B). In addition, none of the FAVG1_07658 flanking genes were closely related to genes flanking the Clade 20 PKS genes. Thus, we consider FAVG1_07658 to be a singlehomolog lineage, which we have designated Clade 68 (Figure 1), and we hypothesize that it catalyzes synthesis of a molecule that is similar in structure to the fusaridione polyketide. Assuming FAVG1_07658 and some of the genes flanking it constitute a biosynthetic gene cluster, we further hypothesize that the corresponding NP is similar to but structurally distinct from fusaridione. Based on current information, however, we cannot rule out the possibility that FAVG1_07658 and Clade 69 PKSs synthesize the same polyketide that is further modified to 
different NPs. Given differences in their sequences, it seems likely that FAVG1_07658 and the Clade 20 PKS homologs give rise to structurally distinct polyketides. Furthermore, given differences in the content of the putative gene clusters in which the PKS genes are located, it is likely that FAVG1_07658 and the Clade 20 PKSs are involved in distinct biosynthetic pathways that give rise to structurally distinct NPs.

The third exception involves orthology group PKS14 described by Hansen et al. (2015), which consisted of a PKS from $F$. avenaceum, $F$. culmorum, $F$. graminearum and $F$. pseudograminearum. In our analysis, however, the $F$. avenaceum PKS, FAVG1_07922, was not resolved in the same clade as the other three PKS14 homologs, but instead was resolved in a clade (Clade 53) with PKSs from F. redolens and F. sacchari. As mentioned previously, all three Clade 53 PKSs are adjacent to an R-PKS present in Clade 48. Thus, our results indicate that PKSs in orthology group PKS14 have been partitioned into two groups: Clade 14 and Clade 53. Clade 14 consists of the $F$. culmorum, $F$. graminearum, and $F$. pseudograminearum $\mathrm{PKSs}$ from orthology group PKS14 as well as F. poae PKS Fpo 93; and Clade 53 consists of FAVG1_07922 and homologs from F. redolens and F. sacchari (Figure 1).

The fourth exception involves orthology groups PKS34 and PKS43. In the Hansen et al. study, group PKS34 consisted of a single PKS from $F$. solani, JGI 123123, and group PKS43 consisted of a PKS from $F$. avenaceum and FIESC 5 ( $F$. equiseti in Hansen et al.). In our analysis, groups PKS34 and PKS43 were resolved in the same clade (Clade 43) along with six other PKS homologs. In addition, all eight PKSs of this clade are flanked by orthologs of the same gene, encoding a putative chalcone synthase, suggesting a two-gene cluster. Because PKS34 described by Hansen et al (2015) was included in Clade 43 in the current study, and in an attempt to avoid confusion, we have not included a Clade 34 in our clade numbering system.

Distribution of PKSs in Fusarium. Examination of the occurrence of the 488 PKS genes in the 31 Fusarium species surveyed revealed multiple and disparate patterns of distribution (Figure 4). At one extreme, homologs of Clades 3,7 and 8 occur in all 31 species. This finding is consistent with previous studies that reported these three PKS genes occurred in all species examined (Brown et al., 2012a; Ma et al., 2010; Wiemann et al., 2013). Homologs in Clade 3 are required for the synthesis of fusarubin (Gaffoor et al., 2005; Studt et al., 2012), while the polyketides and NPs formed via the activity of the PKS homologs in Clades 7 and 8 have not been identified. At 
the other extreme in distribution, 15 PKS clades consist of only one Fusarium homolog, and therefore, such PKSs are unique to one of the 31 Fusarium species examined. For example, Clade 58 consists of only the $F$. aywerte PKS Fay 343, and Clade 59 consists of only the $F$. bulbicola PKS Fbu 946. F. circinatum, F. miscanthi, F. redolens, F. solani f. sp. pisi and F. scirpi each have one of the unique PKSs; F. aywerte, $F$. bulbicola and $F$. thapsinum each have two of the unique PKSs; and F. avenaceum has four unique PKSs. Eight PKS clades consist of two PKS homologs, and seven of these clades include PKSs that occur in species from only one species complex. Furthermore, five of the two-homolog clades (Clades 29, 30, 32, 33, and 35) occur only in the two representative members of the $F$. solani complex: F. solani $\mathrm{f}$. sp. pisi and F. euwallaceae. This restricted distribution may reflect that this complex is relatively distantly related to the other complexes examined (Figure 2) (O'Donnell et al., 2013).

PKSs in some other clades (e.g. Clades 16, 20, 21 and 22) occur widely or even uniformly among members of closely related species complexes. Clade 16 homologs, for example, occur in all species examined from the $F$. babinda, $F$. fujikuroi, $F$. nisikadoi, $F$. oxysporum, and F. redolens complexes as well as in the closely related species Fusarium sp. NRRL 25184 and the two representative species of the F. tricinctum species complex. Although a Clade 16 homolog is present in both members of the $F$. oxysporum complex that were included in the current study (i.e. F. foetens and $F$. oxysporum strain 4287 ), a homolog of the gene was absent in two of 12 strains of F. oxysporum previously examined (Brown and Proctor, 2013). Thus, although our analysis indicated that PKSs from the same Clades occur uniformly among species of one or more species complexes, deeper sampling will likely reveal variation in occurrence within species and species complexes.

PKSs in some clades also occur widely in diverse species but exhibit a discontinuous distribution; that is, they are present in some species complexes or some members of a complex but absent in others. Examples of discontinuously distributed PKSs are those in Clades 2, 5 and 10. PKS homologs that occur in relatively few species also tend to exhibit discontinuous distribution, sometimes across diverse species complexes. PKS homologs in Clade 43, for example, occur in only eight species, but these species are dispersed across six species complexes (Figure 1).

The discontinuous distribution of PKS homologs and other NP biosynthetic genes in Fusarium has been reported previously (Proctor et al., 2013; Wiemann et al., 2013). Multiple 
evolutionary processes, including horizontal transfer and loss/deletion of the genes, have been proposed to contribute to such discontinuity (Proctor et al., 2013; Rooney and Ward, 2005; Slot and Rokas, 2011; Wiemann et al., 2013). Determining the causes of discontinuity of PKS homologs observed in the current study (e.g., those in Clades 2, 5, 10, 15, 43 and 53) will require more detailed phylogenetic analyses of the genes/proteins, including comparison of levels of divergence of PKS genes and housekeeping genes used to infer species phylogenies.

The results of the current and previous studies suggest that recent duplication events have not made a large contribution to variability in the occurrence of PKS genes among Fusarium species. Of the 490 Fusarium PKSs analyzed here, only two, FOXG_15886 and FOXG_14850 (Clade 22), appear to be the result of a recent gene duplication event. These two PKS gene paralogs occur in $F$. oxysporum strain 4287 but not in any of the other $11 F$. oxysporum strains for which genome sequences were available (Brown and Proctor, 2013). These two PKSs exhibit $98 \%$ nucleotide sequence identity. Further analysis in the current study indicated that $F$. commune PKS Fco 3239 is more similar to FOXG_14850 than to FOXG_15886 (Figure 1). One possible explanation for the relationships of these three homologs to one another is that FOXG_14850 is the progenitor homolog and FOXG_15886 was derived from it by a duplication event. Alternatively, FOXG_15886 could have been obtained by horizontal gene transfer from a closely related Fusarium donor. A number of other Clades contain two PKSs from the same Fusarium species but, the sequence identity shared by such paralogs is much lower than the identity shared by FOXG_14850 and FOXG_15886. For example, the F. acuminatum PKSs paralogs, Fac 2643 and Fac 676, which were both resolved within Clade 22, share 64\% nucleotide identity. The lower degree of shared identity indicates that if the presence of these two PKSs in F. acuminatum is the result of duplication, the duplication event was almost certainly more ancient than the putative duplication event leading to the presence of FOXG_14850 and FOXG_15886 in a single strain of $F$. oxysporum. However, as with FOXG_14850 and FOXG_15886, horizontal transfer also provides a possible explanation for the presence of the two relatively closely related PKSs in $F$. acuminatum.

Functional predictions. Clades that include a PKS of known function, either a previously characterized Fusarium PKS or a PKS from another Ascomycete, also support the hypothesis that PKSs in the same clade are involved in the synthesis of the same or structurally similar 
metabolites. Twenty-four clades include at least one PKS for which the polyketide product (or polyketide-derived NP) has been identified (Table 2). The 175 PKSs that make up these 24 clades represent $36 \%$ of the Fusarium PKSs examined. Sixteen clades include a functionally characterized PKS from a Fusarium, while eight clades include a functionally characterized PKS from other Ascomycetes (Table 2).

Our analysis of PKSs indicates that the genetic potential for production of some polyketide-derived metabolites is more widely distributed than previously reported. For example, fusaridione production has been reported only in F. heterosporum (Table 3) (Kakule et al., 2013). However, orthologs of the fusaridione PKS (Clade 69, Figure 1 and Figure 3B) occur in four other species. Likewise, although equisetin production has been previously reported only in a limited number of Fusarium species (Hansen et al., 2015; Kakule et al., 2013), the results of the current study indicate that the equisetin PKS occurs in 18 species (Clade 18, Figure 1). This in turn suggests that a greater diversity of species have the genetic potential to produce equisetin than previously reported.

Clades 17, 44, 45 and 52 include PKS homologs from fungi other than Fusarium and are encoded in proposed biosynthetic gene clusters for which the corresponding polyketide and/or polyketide-derived NP has been identified. Examination of genes flanking the Fusarium PKS genes in these clades revealed the presence of homologs of the cluster genes described in the other fungi (Figure 5). These findings provide evidence for the potential production of NPs that have not yet been reported in Fusarium. For example, the alternapyrone PKS (alt1) from Alternaria solani was resolved as a basal lineage of Clade 52 (Figure 1). In A. solani, the proposed alternapyrone biosynthetic gene cluster consists of five genes (alt1 - alt5) (Fujii et al., 2005). All six fusaria with PKS homologs in Clade 52 also have homologs of alt2, alt3 and alt4, but none have a homolog of alt1. Except for the absence of alt1, the order and orientation of alt genes is the same in Fusarium and Alternaria (Figure 5A). Likewise, the solanapyrone PKS gene (soll) from $A$. solan $i$ was resolved in Clade 44 along with $F$. avenaceum PKS FAVG1_08719 and F. acuminatum PKS Fac 3328. In A. solani, the proposed solanapyrone biosynthetic cluster consists of six genes (soll - sol6) (Kasahara et al., 2010). The region adjacent to FAVG1_08719 and Fac 3328 genes includes homologs of sol2, sol3, sol4, sol5 and sol6 (Figure 5B). 
The Beauveria bassiana tenellin PKS gene, tenS, was resolved as a basal lineage of Clade 45 (Figure 1). The proposed tenellin biosynthetic gene cluster consists of five genes: ORF0, tenA, tenB, tenC, tenS (Figure 5C) (Eley et al., 2007). A homologous cluster responsible for synthesis of the tenellin-like NP fumosorinone has also been characterized in Isaria fumosorosea (Figure 5C) (Liu et al., 2015). The three fusaria with Clade 45 PKSs also have homologs of tenellin cluster genes in addition to the PKS gene, but all lack a tenB homolog (Figure 5C). In addition, the tenA homolog in $F$. poae is located on a different contig than the other ten genes, suggesting fragmentation of the cluster. All three fusaria have two additional genes (designated as $F$ and $M$ for the purposes of this discussion) next to or between the ten genes. We also identified tenellin-like gene cluster in Trichoderma reesei that includes homologs of $F$ and $M$. The location of $F$ and $M$ next to the ten genes in Fusarium and Trichoderma suggests that they are involved in synthesis of a tenellin/fumosorinone-like metabolite in both genera.

The occurrence of homologs of the alternapyrone, solanapyrone and tenellin/ fumosorinone clusters indicates a genetic potential for synthesis of these or structurally similar metabolites that, to our knowledge, have not previously been reported in Fusarium. Because the $F$. avenaceum homolog of the solanapyrone cluster includes homologs of all six sol genes described in A. solani, it is possible that if the cluster were active in $F$. avenaceum, it would result in production of solanapyrone. In contrast, because the gene content of the Fusarium homologs of the tenellin cluster differs from the content in the cluster in B. bassiana, it is possible that the metabolic products of the Fusarium clusters differ in structure from both tenellin and fumosorinone. Differences in gene content of homologous gene clusters among fungal genera can lead to analogs of metabolites that have the same core structure but differ markedly in modifications to the structure, as is the case for the trichothecene biosynthetic gene cluster in Fusarium and Stachybotrys (Brown et al., 2002; Semeiks et al., 2014). However, more subtle differences in production can occur in different fungi even when there are marked differences in gene content of homologous clusters, as observed with the production of different numbers of fumonisin analogs mycotoxins in Aspergillus and Fusarium (Susca et al., 2014).

Five other PKS clades (Clades 55, 64, 54, 17 and 56) also include functionally characterized PKSs from other fungi that provide insight into the functions of the Fusarium PKSs. For example, PKS Clade 55 and 64 each consist of two PKSs: one from $F$. thapsinum and the other from Aspergillus nidulans. In both fungi, and as mentioned previously, the Clade 55 
and 64 PKS genes are located adjacent to one another, and in A. nidulans both PKSs are required for synthesis of the polyketide 2,4-dihydroxy-6-[(3E, 5E, 7E)-2-oxonona-3,5,7trienyl]benzaldehyde (Ahuja et al., 2012). Thus, we propose that the $F$. thapsinum Clade 55 and 64 PKS function in concert during the synthesis of the same NP, as described above for zearalenone: i.e., the Clade 55 R-PKS synthesizes a polyketide that is used as a starter unit for the Clade 64 NR-PKS to synthesis a larger polyketide.

PKS Clade 54 includes a single Fusarium PKS, F. bulbicola Fbu 86 as well as the Acremonium strictum PKS MosA, which is required for synthesis of 3-methylorsellinic acid (Bailey et al., 2007). Based on this close phylogenetic relationship, Fbu 86 is likely involved in the synthesis of 3-methylorsellinic acid or a structurally similar polyketide. PKS Clade 17 includes seven Fusarium PKSs as well as the Alternaria brassicicola PKS Dep5, which is required for depudecin synthesis (Wight et al., 2009). Based on their close phylogenetic relationship to Dep5, these seven Fusarium PKSs likely synthesize the polyketide that serves as the first committed intermediate in the depudecin biosynthetic pathway. PKS Clade 56 includes F. circinatum FCIR_08230, the Alternaria solani PKS (MlnS) required for synthesis of mellein, and the Aspergillus terreus PKS (AtX) required for synthesis of 6-methylsalicylic acid (MSAS). Because FCIR_08230 and MlnS are more closely related to one another than either is to AtX, FCIR_08230 is likely required for synthesis of a polyketide that is more similar in structure to mellein than MSAS.

Non-reducing PKSs and pigments. Many fungal pigments consist of two or more fused aromatic rings that are formed via the activity of an NR-PKS. The NR-PKSs responsible for synthesis of four such Fusarium pigments have been characterized. The four pigments and the corresponding PKS clades resolved in our analysis are: fusarubins - Clade 3 (Studt et al., 2012); aurofusarin - Clade 12 (Gaffoor et al., 2005; Malz et al., 2005); bikaverin - Clade 16 (Linnemannstons et al., 2002); an uncharacterized red pigment of $F$. solani f. sp. pisi-Clade 35 (Graziani et al., 2004). The Fusarium NR-PKSs responsible for pigment production are part of a large well-supported clade (bootstrap value 92) within the NR-PKS group and that includes homologs from other fungi (Figure 1).

The distribution of PKS homologs in Clades 3, 12, 16 and 35 indicates that 28 of the 31 species examined have the genetic potential to produce two or more PKS-derived pigments. The 
fusarubin NR-PKS (Clade 3) is the most wide-spread, being present in all 31 species, while the bikaverin NR-PKS (Clade 16) is present in 22 species across the F. tricinctum, F. babinda, $F$. fujikuroi, F. nisikadoi, F. oxysporum and F. redolens complexes. Of the remaining six species examined, four in the F. sambucinum species complex have a Clade 12 NR-PKS homolog, which is required for aurofusarin synthesis, and two in the $F$. solani species complex have a Clade 35 NR-PKS homolog which is required for synthesis of an uncharacterized red pigment. $F$. acuminatum and $F$. avenaceum, the two representative members of the $F$. tricinctum species complex, are unique in that they have homologs of four pigment biosynthetic NR-PKSs: the fusarubin, aurofusarin, and two bikaverin-like NR-PKSs.

Aromatic pigments produced by Fusarium can impart color to mycelia, perithecia and/or culture media. Bikaverin can impart a reddish color to the mycelia and culture media of fusaria that have PKS orthologs from Clade 16 (Wiemann et al., 2009). Fusarubin can also impart a reddish color to the mycelia and culture media of fungi with a homolog from Clade 3 (Studt et al., 2012). Although the identity of the compound(s) responsible for the dark purple pigmentation of perithecia of the F. fujikuroi, F. sambucinum, and F. tricinctum species complexes has not yet been reported, it is almost certainly a fusarubin analog or derived from a fusarubin(s), because deletion of the NR-PKS gene required for fusarubin production results in colorless perithecia (Brown et al., 2012b; Gaffoor et al., 2005; Proctor et al., 2007; Studt et al., 2012). The two F. solani complex species (F. euwallaceae and F. solani f. sp. pisi) have the fusarubin PKS gene as well as other genes in the fusarubin biosynthetic gene cluster (Proctor et al., 2007; Studt et al., 2012; Wiemann et al., 2009). However, perithecia produced by members in this species complex are red. This pigmentation is most likely imparted by a compound synthesized by the Clade 35 NR-PKSs rather than by the Clade 3 (fusarubin) NR-PKSs. This conclusion is based on the fact that deletion of the Clade 35 NR-PKS gene in F. solani f. sp. pisi results in colorless perithecia (Graziani et al., 2004).

PKS pseudogenes. BLASTn analysis of the 31 Fusarium genome sequences revealed that in addition to the 488 apparently functional PKS genes, the genomes collectively have 81 pseudogenized PKS genes (Table 1 and Supplemental Table 5). In some cases, pseudogenization resulted from single-nucleotide mutations (transitions, transversions, insertions or deletions) that introduced frame shifts and/or internal stop codons into coding regions. In 
other cases, pseudogenization resulted from larger deletions of $10 \mathrm{~s}-1000 \mathrm{~s}$ of nucleotides. The PKS pseudogenes constitute almost $15 \%$ of the combined functional and nonfunctional PKS genes in the species examined. The levels of identity were sufficient to determine the PKS Clades to which $93 \%$ of the pseudogenes were most closely related. This analysis indicates that the pseudogenes were nonfunctional members of 27 Clades from across the NR-PKS and R-PKS groups (Figure 6). Although no pseudogenes were noted in the two Rm-PKS Clades, this could be due to the limited number of PKSs of this type present in Fusarium. The number of PKS pseudogenes per clade ranged from one to 10 (Figure 6), while the number of PKS pseudogenes per species ranged from zero to six (Table 1). The obvious, immediate consequence of pseudogenization of a PKS gene is loss of potential to synthesize the corresponding polyketide, any intermediates, or final NP(s) of the biosynthetic pathway for which the polyketide serves as a precursor. Pseudogenization could also indirectly influence the synthesis of other polyketides because loss of function of one PKS has the potential to increase availability of carboxylic acids used as substrates for polyketide synthesis by other PKSs.

We found two examples where strains of the same species differed in the presence of a functional versus pseudogenized PKS gene. The first example is the $F$. avenaceum $\mathrm{PKS}$ gene FAVG1_08496 (Clade 49). Sequence comparisons of this gene in three F. avenaceum strains indicate that it is functional in strain 05001 but pseudogenized in strains LH03 and LH27. In both strains LH03 and LH27, the pseudogenized FAVG1_08496 is missing nucleotide sequence corresponding to the first 3206 predicted AAs. The second example is the fusarin mycotoxin PKS gene (Clade 10) in F. proliferatum. A sequence comparison indicates that the gene is functional in F. proliferatum strain ITEM 2336 but pseudogenized in strain NRRL 62905. In addition to missing sequence corresponding to the first 2080 predicted AA of the PKS, NRRL 62905 is also missing sequence corresponding to eight other genes that comprise the fusarin biosynthetic gene cluster (Brown et al., 2012b).

PKS Clade 17 has the largest number of pseudogenes. Functional PKSs of this clade are likely involved in synthesis of depudecin, a linear polyketide that was first isolated from Alternaria brassicicola (Isaka et al., 2000) and that inhibits histone deacetylase (Wight et al., 2009). The distribution of functional and pseudogenized homologs of the depudecin PKS gene among members of the $F$. babinda, $F$. chlamydosporum, $F$. fujikuroi, $F$. nisikadoi, $F$. oxysporum and F. redolens species complexes as well as Fusarium sp. NRRL 25184 (Figure 2) suggests that 
559 a functional homolog of the gene was present in the common ancestor of these fusaria and that

560

561

562

563

564

565

566

567

568

569

570

571

572

573

574

575

576

577

578

579

580

581

582

583

584

585

586

587

588

pseudogenization of the gene occurred independently multiple times as species diverged from the ancestor.

Conclusions. In the pre-genomics era, Fusarium served as a rich source of metabolites in numerous NP characterization programs (Cole et al., 2003). Initial genomic studies indicated that fungi have the genetic potential to synthesize many more NPs than expected based on chemical analysis. The results of the current study suggest that the Fusarium species examined have the genetic potential to produce 64 structurally distinct polyketide-derived NPs. Individual species of Fusarium tend to have similar numbers of PKS and nonribosomal peptide synthetase (NRPS) genes (Hansen et al., 2015; Wiemann et al., 2013). Thus, it is likely that the 31 species examined here have the potential to produce a number of structurally distinct nonribosomal peptide-derived NPs that is similar to the number of polyketide-derived NPs that they produce.

Fusarium species also have terpene synthase and prenyltransferase genes, which catalyze synthesis of molecules that serve as core structures for other classes of NPs. However, in individual species, the numbers of these other synthase genes are typically much lower than the numbers of PKS and NRPS genes (Ma et al., 2010; Wiemann et al., 2013). Regardless, mining of genomic sequences of Fusarium for NP biosynthetic genes suggests that this genus has a tremendous capacity to produce a structurally diverse array of NPs and that remarkable variation in production can exist between species and species complexes.

Together, the discontinuous distribution of multiple PKS genes, the presence of multiple PKSs that are unique to a single species or species complex, and the relatively high percentage of PKS pseudogenes indicate that dynamic changes in production of polyketide-derived NPs have occurred during the evolution of Fusarium. Variation in PKS gene content within single species (e.g., the fusarin PKS in F. proliferatum) further suggests that some changes have occurred relatively recently. Most Fusarium isolates are recovered from agricultural plants. Therefore, the variation in Fusarium PKS content observed here and in other studies leads to questions of whether agricultural practices contribute to variation in NP production in this fungus; and further whether understanding how such variation arises could contribute to strategies that reduce crop diseases and mycotoxin contamination problems caused by Fusarium. 
In the current study, we have provided evidence that Fusarium has the genetic potential 590 to produce seven NPs that are the same or structurally similar to NPs reported in other fungi.

591 Thus, this study and others like it (Throckmorton et al., 2015) can guide efforts to identify NPs 592 produced by Fusarium (Chiang et al., 2010; Niehaus et al., 2014; von Bargen et al., 2015) that 593 have the potential to contribute to the ability of the fungus to occupy a wide range of habitats, 594 including a diversity of crop plants.

595

\section{Acknowledgements}

597 We would like to thank Chris McGovern, Stephanie Folmar, Marcie L. Moore and Crystal 598 Probyn for technical assistance and Nathane Orwig for operating the genome sequencing 599 platforms. Mention of trade names or commercial products in this article is solely for the 600 purpose of providing specific information and does not imply recommendation or endorsement 601 by the US Department of Agriculture. USDA is an equal opportunity provider and employer. 602 603 
604

605

606

607

608

609

610

611

612

613

614

615

616

617

618

619

620

621

622

623

624

625

626

627

628

629

630

631

Figure 1. Genealogy of 490 Fusarium PKSs and 55 PKSs from other Ascomycetes inferred from maximum likelihood analysis of nucleotide sequences of PKS coding regions. The NR-PKS and R-PKS I, II and III groups are delineated with red horizontal lines, and the 67 clades are indicated with a unique number (in red text) to the right of the tree. Statistical support for branches within the phylogenetic tree were generated by bootstrap analysis with 500 pseudoreplicates and are indicted by numbers above the internodes (black text). The protein/gene designations for PKSs corresponding to gene model designations at NCBI and predicted for this study are listed in Table 1. The gene name of the 55 PKSs from other Ascomycetes as well as the name of the organism and polyketide/NP are also indicated to the right of the tree. The names of 16 Fusarium metabolites are indicated adjacent to each PKS Clade number. Representative PKSs that have been functionally characterized are indicated in red text. A representative chemical structure for a functionally characterized PKS is indicated for eight Clades that include a Fusarium PKS. In the chemical structures, thick lines represent the region of a structure that is synthesized by a PKS. The Gallus gallus fatty acid synthase genes served as the outgroup.

Figure 2. Species phylogeny of the 31 Fusarium species examined in the current study. The tree was generated by maximum likelihood analysis of the concatenated sequences of the coding regions of 20 housekeeping genes (Supplemental Table 3). Numbers above internodes represent bootstrap support values based on 500 pseudoreplicates. Taxa are color coded according to species complex (O'Donnell et al., 2013). The three subclades within F. fujikuroi species complex are indicated: American, African and Asian. The tree and aligned sequence data from which it was inferred have been submitted to TreeBASE:S18497 (URL: http://purl.org/phylo/treebase/phylows/study/TB2:S18497). The presence (+), absence (--) or pseudogenization $(\Psi)$ of homologs of the depudecin PKS (Clade 17) are indicated to the right. 
632 Figure 3. Comparison of Fusarium genes flanking PKSs in Clade 16 (A) and Clade 20, Clade 68 633 and Clade 69 (B). The relevant portion of the phylogram from Figure 1 is depicted on the left. 634 The arrowheads indicate direction of transcription, and the blue arrows represent cluster genes. 635 Bikaverin cluster genes are labeled BIK1 through BIK6. Putative fusaridione cluster genes are 636 labeled $f s d S, K, D, H, G$ and $C$. Green arrows represent genes with a putative function associated with NP biosynthesis. C6 $=$ transcriptional regulator, mono $=$ monooxygenase, red $=$ reductase, hyd $=$ hydrolase, dhy $=$ dehydrogenase, and mfs = MFS type transporter. Black arrows represent genes with an unknown function or genes generally not associated with NP biosynthesis. The shaded area indicate homologous genes in the different Fusarium species.

Figure 4. Distribution of PKSs among the Fusarium species examined in the current study. The total height of each bar (both black and gray) represents the total number of Clades with the number of PKSs indicated on the horizontal axis. The height of the black portion of each bar

644 represents the number of Clades in which the function of at least one PKS has been determined.

645

646

647

648

649

650

651

652

653

654

655

656

657

658

659

660
Figure 5. Comparison of Fusarium genes flanking PKSs that are closely related homologs of PKSs from other Ascomycetes and for which the polyketide/or polyketide-derived NP has been identified. The arrowheads indicate direction of transcription, and the blue arrows represent cluster genes. A. Alternapyrone cluster homologs. The proposed alternapyrone cluster genes from Alternaria solani are labeled alt1 through alt5. The green arrow represents the alt cluster gene not conserved in Fusarium. B. Solanapyrone cluster homologs. The proposed solanapyrone cluster genes from Alternaria solani are labeled soll through sol6. C. Tenellin cluster homologs. The proposed tenellin cluster genes are labeled tenS, $A, B, C$ and 1 . The orange arrows represent putative genes in Trichoderma reesii and Fusarium not present in the ten cluster in B. bassiana.

Figure 6. Distribution of functional and pseudogenes across PKS Clades. The PKS Clades identified by phylogenetic analysis are indicated on the horizontal axis. The total number of functional (black) and pseudogenes (grey) in each Clade is indicated on the vertical axis. The total number of functional and pseudogenes per PKS Clade is indicated along the horizontal axis. 


\section{References}

Ahuja, M., Chiang, Y. M., Chang, S. L., Praseuth, M. B., Entwistle, R., Sanchez, J. F., Lo, H. C., Yeh, H. H., Oakley, B. R., Wang, C. C., 2012. Illuminating the diversity of aromatic polyketide synthases in Aspergillus nidulans. J. Am. Chem. Soc. 134, 8212-8221.

Bailey, A. M., Cox, R. J., Harley, K., Lazarus, C. M., Simpson, T. J., Skellam, E., 2007. Characterisation of 3-methylorcinaldehyde synthase (MOS) in Acremonium strictum: first observation of a reductive release mechanism during polyketide biosynthesis. Chem. Commun., 4053-4055.

Baker, S. E., Kroken, S., Inderbitzin, P., Asvarak, T., Li, B. Y., Shi, L., Yoder, O. C., Turgeon, B. G., 2006. Two polyketide synthase-encoding genes are required for biosynthesis of the polyketide virulence factor, T-toxin, by Cochliobolus heterostrophus. Mol. PlantMicrobe Interact. 19, 139-149.

Brown, D. W., Busman, M., Proctor, R. H., 2014. Fusarium verticillioides SGE1 is required for full virulence and regulates expression of protein effector and secondary metabolite biosynthetic genes. Mol. Plant Microbe. Interact. 27, 809-823.

Brown, D. W., Butchko, R. A., Baker, S. E., Proctor, R. H., 2012a. Phylogenomic and functional domain analysis of polyketide synthases in Fusarium. Fungal Biol. 116, 318-331.

Brown, D. W., Butchko, R. A., Busman, M., Proctor, R. H., 2012b. Identification of gene clusters associated with fusaric acid, fusarin, and perithecial pigment production in Fusarium verticillioides. Fungal Genet. Biol. 49, 521-532.

Brown, D. W., Lee, S. H., Kim, L. H., Ryu, J. G., Lee, S., Seo, Y., Kim, Y. H., Busman, M., Yun, S. H., Proctor, R. H., Lee, T., 2015. Identification of a 12-gene fusaric acid biosynthetic gene cluster in Fusarium species through comparative and functional genomics. Mol. Plant-Microbe Interact. 28, 319-332.

Brown, D. W., McCormick, S. P., Alexander, N. J., Proctor, R. H., Desjardins, A. E., 2002. Inactivation of a cytochrome P-450 is a determinant of trichothecene diversity in Fusarium species. Fungal Genet. Biol. 36, 224-233.

Brown, D. W., Proctor, R. H., Diversity of polyketide synthases in Fusarium. In: D. W. Brown, R. H. Proctor, Eds.), Fusarium Genomics, Molecular and Cellular Biology. Caister Academic Press, Norfolk, UK, 2013, pp. 143-164.

Brown, D. W., Yu, J.-H., Kelkar, H. S., Fernandes, M., Nesbitt, T. C., Keller, N. P., Adams, T. H., Leonard, T. J., 1996. Twenty-five co-regulated transcripts define a sterigmatocystin gene cluster in Aspergillus nidulans. Proc. Natl. Acad. Sci. U.S.A. 93, 1418-1422.

Campbell, M. A., Rokas, A., Slot, J. C., 2012. Horizontal transfer and death of a fungal secondary metabolic gene cluster. Genome Biol. Evol. 4, 289-293.

Chiang, Y. M., Oakley, B. R., Keller, N. P., Wang, C. C., 2010. Unraveling polyketide synthesis in members of the genus Aspergillus. Appl. Microbiol. Biotechnol. 86, 1719-1736.

Cole, R. J., Jarvis, B. B., Schweikert, M. A., 2003. Handbook of secondary fungal metabolites. Academic Press, San Diego.

Connolly, L. R., Smith, K. M., Freitag, M., 2013. The Fusarium graminearum histone H3 K27 methyltransferase KMT6 regulates development and expression of secondary metabolite gene clusters. PLoS Genet. 9, e1003916.

Cuomo, C. A., Guldener, U., Xu, J. R., Trail, F., Turgeon, B. G., Di Pietro, A., Walton, J. D., Ma, L. J., Baker, S. E., Rep, M., Adam, G., Antoniw, J., Baldwin, T., Calvo, S., Chang, 
Y. L., Decaprio, D., Gale, L. R., Gnerre, S., Goswami, R. S., Hammond-Kosack, K., Harris, L. J., Hilburn, K., Kennell, J. C., Kroken, S., Magnuson, J. K., Mannhaupt, G., Mauceli, E., Mewes, H. W., Mitterbauer, R., Muehlbauer, G., Munsterkotter, M., Nelson, D., O'Donnell, K., Ouellet, T., Qi, W., Quesneville, H., Roncero, M. I., Seong, K. Y., Tetko, I. V., Urban, M., Waalwijk, C., Ward, T. J., Yao, J., Birren, B. W., Kistler, H. C., 2007. The Fusarium graminearum genome reveals a link between localized polymorphism and pathogen specialization. Science. 317, 1400-1402.

Desjardins, A. E., 2006. Fusarium mycotoxins chemistry, genetics and biology. APS Press, St. Paul, MN.

Ehrlich, K. C., Mack, B. M., 2014. Comparison of expression of secondary metabolite biosynthesis cluster genes in Aspergillus flavus, A. parasiticus, and A. oryzae. Toxins. 6, 1916-1928.

Eley, K. L., Halo, L. M., Song, Z., Powles, H., Cox, R. J., Bailey, A. M., Lazarus, C. M., Simpson, T. J., 2007. Biosynthesis of the 2-pyridone tenellin in the insect pathogenic fungus Beauveria bassiana. Chembiochem. 8, 289-297.

Fujii, I., Yoshida, N., Shimomaki, S., Oikawa, H., Ebizuka, Y., 2005. An iterative type I polyketide synthase PKSN catalyzes synthesis of the decaketide alternapyrone with regio-specific octa-methylation. Chem. Biol. 12, 1301-1309.

Gaffoor, I., Brown, D. W., Plattner, R., Proctor, R. H., Qi, W., Trail, F., 2005. Functional analysis of the polyketide synthase genes in the filamentous fungus Gibberella zeae (anamorph Fusarium graminearum). Eukaryotic Cell. 4, 1926-1933.

Gaffoor, I., Trail, F., 2006. Characterization of two polyketide synthase genes involved in zearalenone biosynthesis in Gibberella zeae. Appl. Environ. Microbiol. 72, 1793-1799.

Geiser, D. M., Aoki, T., Bacon, C. W., Baker, S. E., Bhattacharyya, M. K., Brandt, M. E., Brown, D. W., Burgess, L. W., Chulze, S., Coleman, J. J., Correll, J. C., Covert, S. F., Crous, P. W., Cuomo, C. A., De Hoog, G. S., Di Pietro, A., Elmer, W. H., Epstein, L., Frandsen, R. J., Freeman, S., Gagkaeva, T., Glenn, A. E., Gordon, T. R., Gregory, N. F., Hammond-Kosack, K. E., Hanson, L. E., Jimenez-Gasco Mdel, M., Kang, S., Kistler, H. C., Kuldau, G. A., Leslie, J. F., Logrieco, A., Lu, G., Lysoe, E., Ma, L. J., McCormick, S. P., Migheli, Q., Moretti, A., Munaut, F., O'Donnell, K., Pfenning, L., Ploetz, R. C., Proctor, R. H., Rehner, S. A., Robert, V. A., Rooney, A. P., Bin Salleh, B., Scandiani, M. M., Scauflaire, J., Short, D. P., Steenkamp, E., Suga, H., Summerell, B. A., Sutton, D. A., Thrane, U., Trail, F., Van Diepeningen, A., Vanetten, H. D., Viljoen, A., Waalwijk, C., Ward, T. J., Wingfield, M. J., Xu, J. R., Yang, X. B., Yli-Mattila, T., Zhang, N., 2013. One fungus, one name: defining the genus Fusarium in a scientifically robust way that preserves longstanding use. Phytopathology. 103, 400-408.

Georgianna, D. R., Fedorova, N. D., Burroughs, J. L., Dolezal, A. L., Bok, J. W., HorowitzBrown, S., Woloshuk, C. P., Yu, J., Keller, N. P., Payne, G. A., 2010. Beyond aflatoxin: four distinct expression patterns and functional roles associated with Aspergillus flavus secondary metabolism gene clusters. Mol. Plant Pathol. 11, 213-226.

Graziani, S., Vasnier, C., Daboussi, M. J., 2004. Novel polyketide synthase from Nectria haematococca. Appl. Environ. Microbiol. 70, 2984-2988.

Hansen, F. T., Gardiner, D. M., Lysoe, E., Fuertes, P. R., Tudzynski, B., Wiemann, P., Sondergaard, T. E., Giese, H., Brodersen, D. E., Sorensen, J. L., 2015. An update to polyketide synthase and non-ribosomal synthetase genes and nomenclature in Fusarium. Fungal Genet. Biol. 75, 20-29. 
Hansen, F. T., Sorensen, J. L., Giese, H., Sondergaard, T. E., Frandsen, R. J., 2012. Quick guide to polyketide synthase and nonribosomal synthetase genes in Fusarium. Int. J. Food Microbiol. 155, 128-136.

Hopwood, D. A., Khosla, C., Genes for polyketide secondary metabolic pathways in microorganisms and plants. In: D. J. Chadwick, J. Whelan, Eds.), Secondary Metabolites: Their Function and Evolution. John Wiley and Sons, Chichester, UK, 1992, pp. 88-112.

Isaka, M., Jaturapat, A., Kladwang, W., Punya, J., Lertwerawat, Y., Tanticharoen, M., Thebtaranonth, Y., 2000. Antiplasmodial compounds from the wood-decayed fungus Xylaria sp. BCC 1067. Planta Med. 66, 473-475.

Jorgensen, S. H., Frandsen, R. J., Nielsen, K. F., Lysoe, E., Sondergaard, T. E., Wimmer, R., Giese, H., Sorensen, J. L., 2014. Fusarium graminearum PKS14 is involved in orsellinic acid and orcinol synthesis. Fungal Genet. Biol. 70, 24-31.

Kakule, T. B., Sardar, D., Lin, Z., Schmidt, E. W., 2013. Two related pyrrolidinedione synthetase loci in Fusarium heterosporum ATCC 74349 produce divergent metabolites. Chem. Biol. 8, 1549-1557.

Kasahara, K., Miyamoto, T., Fujimoto, T., Oguri, H., Tokiwano, T., Oikawa, H., Ebizuka, Y., Fujii, I., 2010. Solanapyrone synthase, a possible Diels-Alderase and iterative type I polyketide synthase encoded in a biosynthetic gene cluster from Alternaria solani. ChemBioChem. 11, 1245-1252.

Keller, N. P., 2015. Translating biosynthetic gene clusters into fungal armor and weaponry. Nat. Chem. Biol. 11, 671-677.

Keller, N. P., Turner, G., Bennett, J. W., 2005. Fungal secondary metabolism - from biochemistry to genomics. Nat. Rev. Microbiol. 3, 937-947.

Kim, Y. T., Lee, Y. R., Jin, J., Han, K. H., Kim, H., Kim, J. C., Lee, T., Yun, S. H., Lee, Y. W., 2005. Two different polyketide synthase genes are required for synthesis of zearalenone in Gibberella zeae. Mol. Microbiol. 58, 1102-1113.

Kroken, S., Glass, N. L., Taylor, J. W., Yoder, O. C., Turgeon, B. G., 2003. Phylogenomic analysis of type I polyketide synthase genes in pathogenic and saprobic ascomycetes. Proc. Natl. Acad. Sci. 100, 15670-15675.

Leslie, J. F., Summerell, B. A., 2006. The Fusarium laboratory manual. Blackwell Publishing, Ames, Iowa.

Linnemannstons, P., Schulte, J., del Mar Prado, M., Proctor, R. H., Avalos, J., Tudzynski, B., 2002. The polyketide synthase gene $p k s 4$ from Gibberella fujikuroi encodes a key enzyme in the biosynthesis of the red pigment bikaverin. Fungal Genet. Biol. 37, 134148.

Liu, L., Zhang, J., Chen, C., Teng, J., Wang, C., Luo, D., 2015. Structure and biosynthesis of fumosorinone, a new protein tyrosine phosphatase 1B inhibitor firstly isolated from the entomogenous fungus Isaria fumosorosea. Fungal Genet. Biol. 81, 191-200.

Ma, L. J., van der Does, H. C., Borkovich, K. A., Coleman, J. J., Daboussi, M. J., Di Pietro, A., Dufresne, M., Freitag, M., Grabherr, M., Henrissat, B., Houterman, P. M., Kang, S., Shim, W. B., Woloshuk, C., Xie, X., Xu, J. R., Antoniw, J., Baker, S. E., Bluhm, B. H., Breakspear, A., Brown, D. W., Butchko, R. A. E., Chapman, S., Coulson, R., Coutinho, P. M., Danchin, E. G., Diener, A., Gale, L. R., Gardiner, D. M., Goff, S., HammondKosack, K. E., Hilburn, K., Hua-Van, A., Jonkers, W., Kazan, K., Kodira, C. D., Koehrsen, M., Kumar, L., Lee, Y. H., Li, L., Manners, J. M., Miranda-Saavedra, D., Mukherjee, M., Park, G., Park, J., Park, S. Y., Proctor, R. H., Regev, A., Ruiz-Roldan, M. 
C., Sain, D., Sakthikumar, S., Sykes, S., Schwartz, D. C., Turgeon, B. G., Wapinski, I., Yoder, O., Young, S., Zeng, Q., Zhou, S., Galagan, J., Cuomo, C. A., Kistler, H. C., Rep, M., 2010. Comparative genomics reveals mobile pathogenicity chromosomes in Fusarium. Nature. 464, 367-373.

Malz, S., Grell, M. N., Thrane, C., Maier, F. J., Rosager, P., Felk, A., Albertsen, K. S., Salomon, S., Bohn, L., Schafer, W., Giese, H., 2005. Identification of a gene cluster responsible for the biosynthesis of aurofusarin in the Fusarium graminearum species complex. Fungal Genet. Biol. 42, 420-433.

Morgavi, D. P., Riley, R. T., 2007. An historical overview of field disease outbreaks known or suspected to be caused by consumption of feeds contaminated with Fusarium toxins. Animal Feed Sci. Tech. 137, 201-212.

Niehaus, E. M., Janevska, S., von Bargen, K. W., Sieber, C. M., Harrer, H., Humpf, H. U., Tudzynski, B., 2014. Apicidin F: characterization and genetic manipulation of a new secondary metabolite gene cluster in the rice pathogen Fusarium fujikuroi. PLoS One. 9, e103336.

O'Donnell, K., Rooney, A. P., Proctor, R. H., Brown, D. W., McCormick, S. P., Ward, T. J., Frandsen, R. J., Lysoe, E., Rehner, S. A., Aoki, T., Robert, V. A., Crous, P. W., Groenewald, J. Z., Kang, S., Geiser, D. M., 2013. Phylogenetic analyses of RPB1 and $R P B 2$ support a middle Cretaceous origin for a clade comprising all agriculturally and medically important fusaria. Fungal Genet. Biol. 52, 20-31.

Proctor, R. H., Butchko, R. A., Brown, D. W., Moretti, A., 2007. Functional characterization, sequence comparisons and distribution of a polyketide synthase gene required for perithecial pigmentation in some Fusarium species. Food Addit. Contam. 24, 1076-1087.

Proctor, R. H., Desjardins, A. E., Plattner, R. D., Hohn, T. M., 1999. A polyketide synthase gene required for biosynthesis of fumonisin mycotoxins in Gibberella fujikuroi mating population A. Fungal Genet. Biol. 27, 100-112.

Proctor, R. H., Van Hove, F., Susca, A., Stea, G., Busman, M., van der Lee, T., Waalwijk, C., Moretti, A., Ward, T. J., 2013. Birth, death and horizontal transfer of the fumonisin biosynthetic gene cluster during the evolutionary diversification of Fusarium. Mol. Microbiol. 90, 290-306.

Riley, R., Salamov, A. A., Brown, D. W., Nagy, L. G., Floudas, D., Held, B. W., Levasseur, A., Lombard, V., Morin, E., Otillar, R., Lindquist, E. A., Sun, H., LaButti, K. M., Schmutz, J., Jabbour, D., Luo, H., Baker, S. E., Pisabarro, A. G., Walton, J. D., Blanchette, R. A., Henrissat, B., Martin, F., Cullen, D., Hibbett, D. S., Grigoriev, I. V., 2014. Extensive sampling of basidiomycete genomes demonstrates inadequacy of the white-rot/brown-rot paradigm for wood decay fungi. Proc. Natl. Acad. Sci. USA. 111, 9923-9928.

Rooney, A. P., Ward, T. J., 2005. Evolution of a large ribosomal RNA multigene family in filamentous fungi: birth and death of a concerted evolution paradigm. Proc. Natl. Acad. Sci. USA. 102, 5084-5089.

Schumacher, J., Gautier, A., Morgant, G., Studt, L., Ducrot, P. H., Le Pecheur, P., Azeddine, S., Fillinger, S., Leroux, P., Tudzynski, B., Viaud, M., 2013. A functional bikaverin biosynthesis gene cluster in rare strains of Botrytis cinerea is positively controlled by VELVET. PLoS One. 8, e53729.

Semeiks, J., Borek, D., Otwinowski, Z., Grishin, N. V., 2014. Comparative genome sequencing reveals chemotype-specific gene clusters in the toxigenic black mold Stachybotrys. BMC Genomics. 15, 590. 
Slot, J. C., Rokas, A., 2011. Horizontal transfer of a large and highly toxic secondary metabolic gene cluster between fungi. Curr. Biol. 21, 134-139.

Sorensen, J. L., Sondergaard, T. E., Covarelli, L., Fuertes, P. R., Hansen, F. T., Frandsen, R. J., Saei, W., Lukassen, M. B., Wimmer, R., Nielsen, K. F., Gardiner, D. M., Giese, H., 2014. Identification of the biosynthetic gene clusters for the lipopeptides Fusaristatin A and W493 B in Fusarium graminearum and F. pseudograminearum. J. Nat. Prod. 77, 26192625.

Studt, L., Wiemann, P., Kleigrewe, K., Humpf, H. U., Tudzynski, B., 2012. Biosynthesis of fusarubins accounts for pigmentation of Fusarium fujikuroi perithecia. Appl. Environ. Micro. 78, 4468-4480.

Susca, A., Proctor, R. H., Butchko, R. A., Haidukowski, M., Stea, G., Logrieco, A., Moretti, A., 2014. Variation in the fumonisin biosynthetic gene cluster in fumonisin-producing and nonproducing black aspergilli. Fungal Genet. Biol. 73, 39-52.

Tamura, K., Nei, M., 1993. Estimation of the number of nucleotide substitutions in the control region of mitochondrial DNA in humans and chimpanzees. Mol. Biol. Evol. 10, 512-526.

Tamura, K., Stecher, G., Peterson, D., Filipski, A., Kumar, S., 2013. MEGA6: Molecular Evolutionary Genetics Analysis version 6.0. Mol. Biol. Evol. 30, 2725-2729.

Throckmorton, K., Wiemann, P., Keller, N. P., 2015. Evolution of chemical diversity in a group of non-reduced polyketide gene clusters: using phylogenetics to inform the search for novel fungal natural products. Toxins. 7, 3572-3607.

Vesonder, R. F., P., G., Metabolites of Fusarium. In: J. Chełkowski, (Ed.), Fusarium. Mycotoxins, taxonomy and pathogenicity. Elsevier, Amsterdam, 1989, pp. 492.

von Bargen, K. W., Niehaus, E. M., Krug, I., Bergander, K., Wurthwein, E. U., Tudzynski, B., Humpf, H. U., 2015. Isolation and structure elucidation of Fujikurins A-D: products of the PKS19 gene cluster in Fusarium fujikuroi. J. Nat. Prod. 78, 1809-1815.

Wiemann, P., Keller, N. P., 2014. Strategies for mining fungal natural products. J. Ind. Microbiol. Biotechnol. 41, 301-313.

Wiemann, P., Sieber, C. M., von Bargen, K. W., Studt, L., Niehaus, E. M., Espino, J. J., Huss, K., Michielse, C. B., Albermann, S., Wagner, D., Bergner, S. V., Connolly, L. R., Fischer, A., Reuter, G., Kleigrewe, K., Bald, T., Wingfield, B. D., Ophir, R., Freeman, S., Hippler, M., Smith, K. M., Brown, D. W., Proctor, R. H., Munsterkotter, M., Freitag, M., Humpf, H. U., Guldener, U., Tudzynski, B., 2013. Deciphering the cryptic genome: genome-wide analyses of the rice pathogen Fusarium fujikuroi reveal complex regulation of secondary metabolism and novel metabolites. PLoS Pathog. 9, e1003475.

Wiemann, P., Willmann, A., Straeten, M., Kleigrewe, K., Beyer, M., Humpf, H. U., Tudzynski, B., 2009. Biosynthesis of the red pigment bikaverin in Fusarium fujikuroi: genes, their function and regulation. Mol. Microbiol. 72, 931-946.

Wight, W. D., Kim, K. H., Lawrence, C. B., Walton, J. D., 2009. Biosynthesis and role in virulence of the histone deacetylase inhibitor depudecin from Alternaria brassicicola. Mol. Plant-Microbe Interact. 22, 1258-1267.

Wu, F., 2007. Measuring the economic impacts of Fusarium toxins in animal feeds. Animal Feed Sci. Tech. 137, 363-374.

Zhang, Z., Schaffer, A. A., Miller, W., Madden, T. L., Lipman, D. J., Koonin, E. V., Altschul, S. F., 1998. Protein sequence similarity searches using patterns as seeds. Nucleic Acids Res. 26, 3986-3990. 
Table 1. Information on strains, species and species complexes of Fusarium, PKS gene/enzyme designations, numbers of PKS genes and pseudogenes per species, and sources of genome sequence data used in this study.

\begin{tabular}{|c|c|c|c|c|c|c|}
\hline Fusarium species & Strain & Species complex $^{\mathrm{a}}$ & $\begin{array}{c}\text { PKS } \\
\text { Designation }^{\mathrm{x}} \\
\end{array}$ & PKSs & Pseudo $^{\mathrm{b}}$ & $\begin{array}{c}\text { Sequence } \\
\text { Data }^{c}\end{array}$ \\
\hline F. graminearum & $\mathrm{PH}-1$ and $\mathrm{CS} 3005^{\mathrm{d}}$ & F. sambucinum & FGSG \# & 16 & 0 & NCBI \\
\hline F. pseudograminearum & CS3069 & F. sambucinum & FPSE \# & 14 & 0 & NCBI \\
\hline F. poae & NRRL 26941 & F. sambucinum & Fpo \# & 11 & 2 & this study $^{\mathrm{I}}$ \\
\hline F. culmorum & CS7071 & F. sambucinum & Fcu \# & 13 & 1 & NCBI \\
\hline F. aywerte & NRRL 25410 & F. chlamydosporum & Fay \# & 16 & 4 & this study ${ }^{\mathrm{I}}$ \\
\hline F. scirpi & NRRL 06979 & F. incarnatum-equiseti & Fsc \# & 11 & 3 & this study ${ }^{\mathrm{M}}$ \\
\hline FIESC $5^{\mathrm{e}}$ & CS3069 & F. incarnatum-equiseti & Fsp3 \# & 11 & 2 & NCBI \\
\hline F. acuminatum & CS5907 & F. tricinctum & Fac \# & 15 & 5 & NCBI \\
\hline F. avenaceum & $\mathrm{Fa05001}$ and FaLH27 ${ }^{\mathrm{f}}$ & F. tricinctum & FAVG1 $\#^{g}$ & 27 & 2 & NCBI \\
\hline F. sacchari & FRC M-6865 & F. fujikuroi $(\mathrm{As})^{\mathrm{h}}$ & Fsa \# & 20 & 0 & this study ${ }^{\mathrm{B}}$ \\
\hline F. fujikuroi & IMI58289 & F. fujikuroi (As) & FFUJ \# & 17 & 1 & NCBI \\
\hline F. proliferatum & NRRL 62905 & F. fujikuroi (As) & Fpr \# & 19 & 2 & this study ${ }^{\mathrm{B}}$ \\
\hline F. verticillioides & FRC M-3125 & F. fujikuroi (Af) & FVEG \# & 16 & 0 & NCBI \\
\hline F. thapsinum & FRC M-6563 & F. fujikuroi (Af) & Fth \# & 16 & 3 & this study ${ }^{\mathrm{B}}$ \\
\hline F. dlaminii & NRRL 13164 & F. fujikuroi (Af) & Fdl \# & 16 & 5 & this study ${ }^{\mathrm{B}}$ \\
\hline F. circinatum & Fsp34 & F. fujikuroi (Am) & FCIR \# & 15 & 3 & NCBI \\
\hline F. succisae & NRRL 13298 & F. fujikuroi (Am) & Fsuc \# & 15 & 3 & this study ${ }^{\mathrm{B}}$ \\
\hline F. subglutinans & NSM 107 & F. fujikuroi (Am) & Fsub \# & 18 & 2 & this study ${ }^{\mathrm{B}}$ \\
\hline F. anthophilum & NRRL 25214 & F. fujikuroi (Am) & Fan \# & 19 & 3 & this study $^{\mathrm{I}}$ \\
\hline F. bulbicola & NRRL 13618 & F. fujikuroi (Am) & Fbu \# & 16 & 3 & this study ${ }^{\mathrm{I}}$ \\
\hline Fusarium sp. 52700 & NRRL 52700 & F. fujikuroi & Fsp5 \# & 15 & 6 & this study ${ }^{\mathrm{B}}$ \\
\hline F. miscanthi & NRRL 26231 & F. nisikadoi & Fmi \# & 16 & 3 & this study ${ }^{\mathrm{I}}$ \\
\hline F. gaditjirri & NRRL 45417 & F. nisikadoi & Fga \# & 14 & 6 & this study ${ }^{\mathrm{I}}$ \\
\hline F. commune & NRRL 28387 & F. nisikadoi & Fco \# & 15 & 6 & this study ${ }^{I}$ \\
\hline F. oxysporum & Fol4287 & F. oxysporum & FOXG \# & 17 & 0 & NCBI \\
\hline F. foetens & NRRL 38302 & F. oxysporum & Ffo \# & 14 & 3 & this study ${ }^{\mathrm{I}}$ \\
\hline Fusarium sp. 25184 & NRRL 25184 & not specific & Fsp2 \# & 19 & 3 & this study ${ }^{\mathrm{I}}$ \\
\hline F. redolens & NRRL 22901 & F. redolens & Fre \# & 18 & 4 & this study ${ }^{\mathrm{I}}$ \\
\hline F. babinda & NRRL 25539 & F. babinda & Fba \# & 15 & 4 & this study ${ }^{\mathrm{I}}$ \\
\hline F. solani f. sp. pisi & FGSC 9596 & F. solani & JGI \# & 13 & 1 & NCBI \\
\hline F. euwallaceae & ATTC 74349 & F. solani & Feu \# & 11 & 1 & this study ${ }^{\mathrm{I}}$ \\
\hline F. heterosporum & & F. heterosporum & $f_{s d S}$ and eqxS & 2 & - & NCBI \\
\hline Total & $31^{\mathrm{j}}$ & & & 490 & 81 & \\
\hline
\end{tabular}

${ }^{\text {a }}$ Species complex as described by O’Donnell et al. (2013) and Geiser et al. (2013).

${ }^{x}$ See Supplemental Table 2 for PKS GenBank Accessions and Gene Designations.

${ }^{\mathrm{b}}$ Pseudo refers to pseudogenes or remnant PKSs.

${ }^{\mathrm{c}}$ NCBI: National Center for Biotechnology Information. The I and M superscripts indicate that sequencing was performed at NCAUR using the Ion-Torrent $\left({ }^{\mathrm{I}}\right)$ or Illumina MiSeq $\left({ }^{\mathrm{M}}\right)$ platforms. The B superscript $\left({ }^{\mathrm{B}}\right)$ indicates that sequencing was performed by the Beijing Genome Institute-Hong Kong. See Supplemental Table X for GenBank Accession numbers for 317 new Fusarium PKSs and designations for all of the other PKSs examined.

${ }^{\mathrm{d}}$ F. graminearum strain CS3005 has a PKS that is not present in strain PH-1.

${ }^{\mathrm{e}}$ In Hansen et al. (2015), strain CS3069 is referred to as $F$. equiseti. In the NCBI database, however, this strain is referred to as FIESC 5: i.e., multilocus sequence type 5 of the $F$. incarnatum-equiseti species complex. Our analysis also indicated that strain CS3069 is 
Table 2. Clades of Fusarium PKSs and corresponding polyketides or polyketide-derived NPs identified in Fusarium or Ascomycetes other than Fusarium.

\begin{tabular}{|c|c|c|c|}
\hline Clade \# & $\mathbf{N P}$ & \begin{tabular}{|l|} 
No. PKS \\
homologs
\end{tabular} & Reference \\
\hline \multicolumn{4}{|c|}{ Polyketide/NP identified in Fusarium } \\
\hline 3 & fusarubins & 31 & Studt et al., 2012 \\
\hline 35 & perithecial pigment & 2 & Gaffoor et al., 2005 \\
\hline 12 & aurofusarin & 6 & Gaffoor et al., 2005 \\
\hline 16 & bikaverin & 24 & Wiemann et al., 2009 \\
\hline $13 / 4^{b}$ & zearalenone & $4 / 4$ & Gaffoor and Trail, 2006 \\
\hline 14 & orsellinic acid & 4 & Jorgensen et al., 2014 \\
\hline 24 & fumonisins & 5 & Proctor et al., 1999 \\
\hline 21 & fusaric acid & 21 & Brown et al., 2012 \\
\hline 6 & fusaristatin & 4 & Sorensen et al., 2014 \\
\hline 40 & W493 B & 3 & Sorensen et al., 2014 \\
\hline 9 & fusarielins & 3 & Sorensen et al., 2012 \\
\hline 69 & fusaridione & 4 & Kakule et al., 2013 \\
\hline 18 & equisetin & 18 & Kakule et al., 2013 \\
\hline 39 & fujikurin & 3 & von Bargen et al., 2015 \\
\hline 10 & fusarins & 18 & Song et al., 2004 \\
\hline \multicolumn{4}{|c|}{ Polyketide/NP identified in Ascomycete other than Fusarium } \\
\hline 54 & 3-methylorsellinic acid & 1 & Bailey et al., 2007 \\
\hline $55 / 64^{\mathbf{b}}$ & oxononal benzaldehyde & $1 / 1$ & Ahuja et al., 2012 \\
\hline 56 & mellein & 1 & Chooi et al., 2015 \\
\hline 17 & depudecin & 7 & Wight et al., 2009 \\
\hline 44 & solanapyrone & 1 & Kasahara et al., 2010 \\
\hline 52 & alternapyrone & 6 & Fujii et al., 2005 \\
\hline 45 & tenellin/fumosorinone & 3 & Eley et al., 2007; Liu et al.,2015 \\
\hline
\end{tabular}

${ }^{a}$ Number of PKS homologs in the clade. 4/4 and 1/1 indicate the number of PKS homologs in the two clades required for synthesis of zearalenone and oxononal benzaldehyde respectively.

b Two PKSs are involved or likely involved in synthesis of NP. 
most closely related to FIESC 5 (data not shown). In the current study, we refer to strain CS3069 as FIESC 5, because FIESC 5 and F. equiseti are not equivalent (O'Donnell et al., 2009).

${ }^{\mathrm{f}}$ F. avenaceum strain FaLH27 has a PKS that is not present in strain Fa05001.

${ }^{\mathrm{g}}$ FAVG1 \#s refer to $24 \mathrm{~F}$. avenaceum PKS gene names at NCBI. We identified three additional PKSs, labeled Faven\#, in the $F$. avenaceum genomic DNA in the NCBI database that were not assigned FAVG1 \#s.

${ }^{\mathrm{h}}$ The $F$. fujikuroi species complex includes three clades: Asian (As), African (Af) and American (Am).

${ }^{\mathrm{i}} 14$ F. oxysporum PKS were retrieved from strain Fol4287. One PKS each was retrieved strain Fo5176, FOSC 3-a and Fo47.

${ }^{\mathrm{j}}$ This value refers to the number of Fusarium genomes screened for PKS genes and pseudogenes. The genome of $F$. heterosporum was not examined in this study. The two $F$. heterosporum PKSs examined were recovered from the NCBI database. 
Figure 1

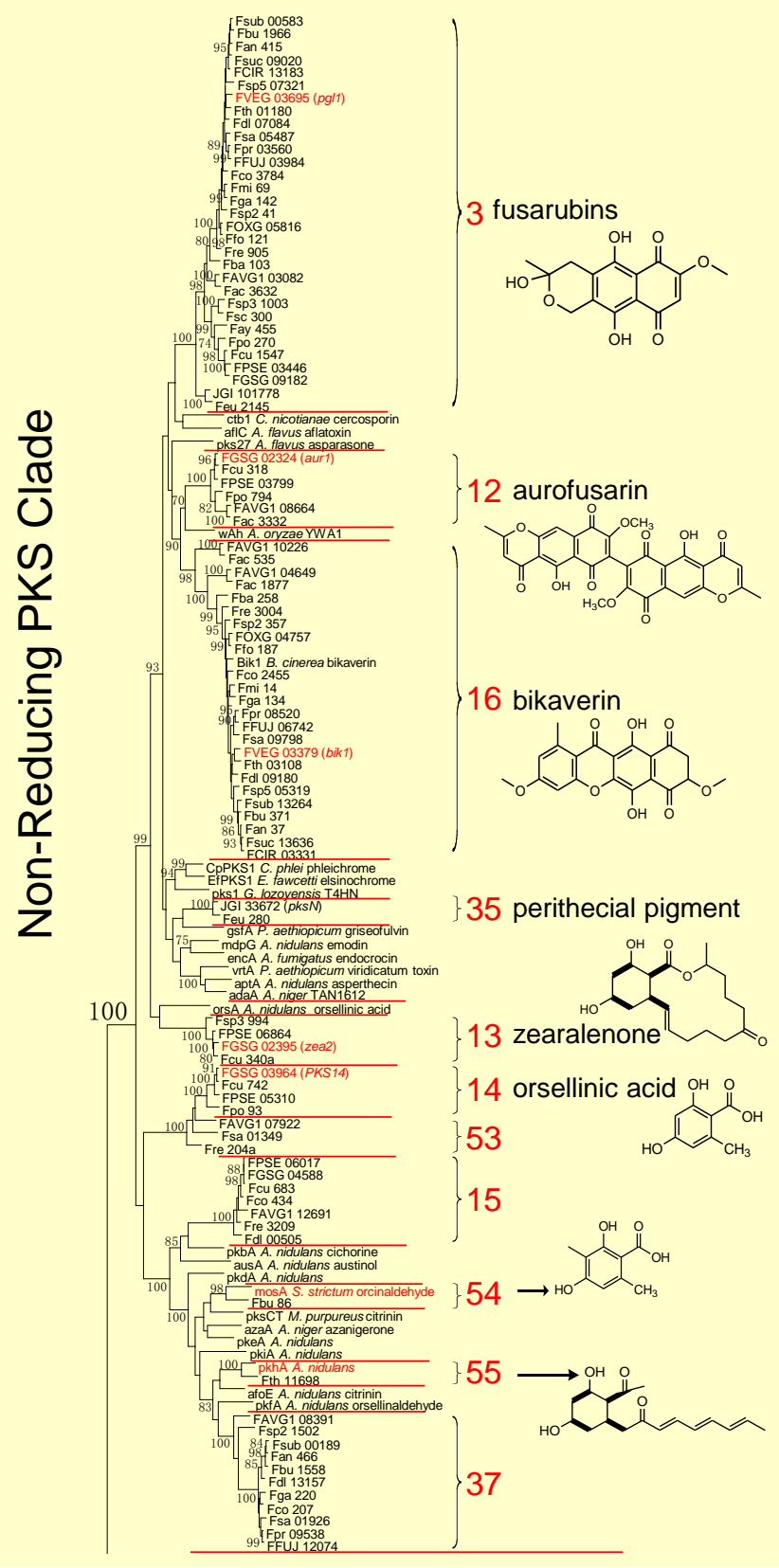

Figure 1. Legend
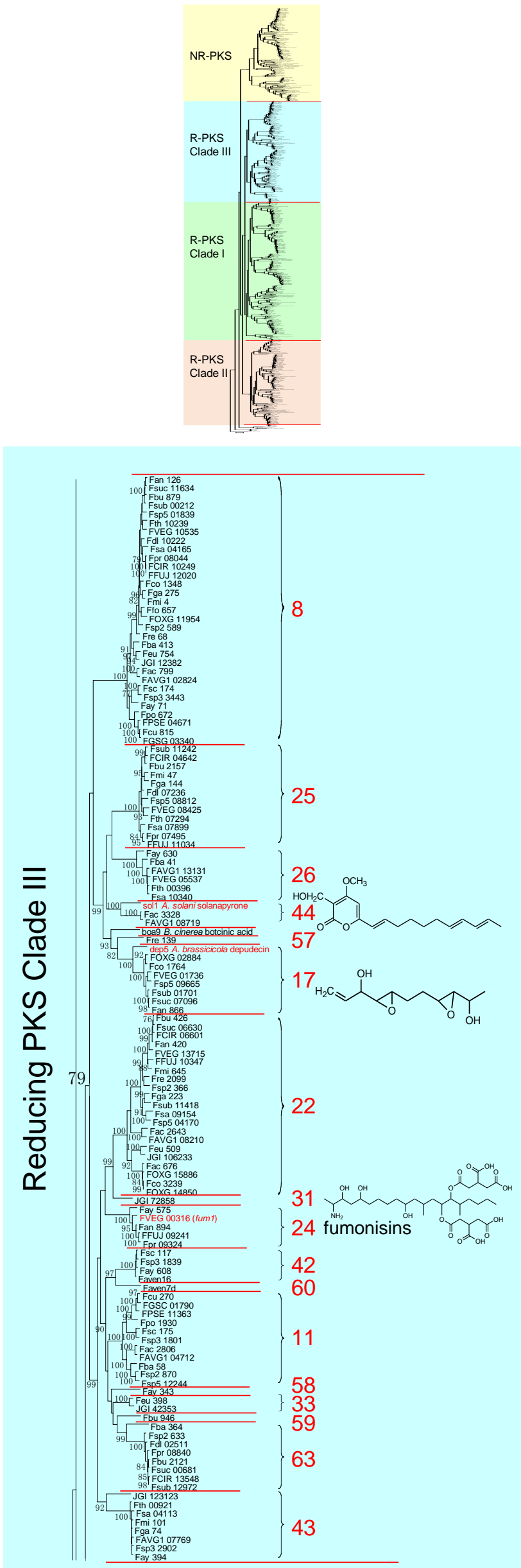
Figure 1. Continued

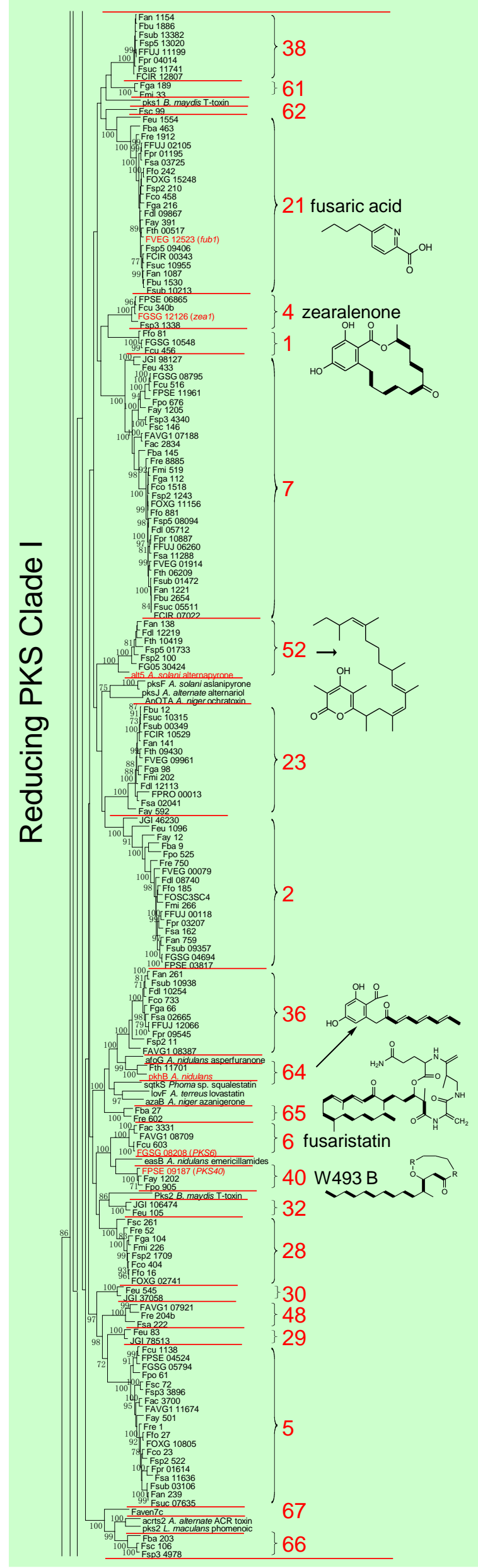

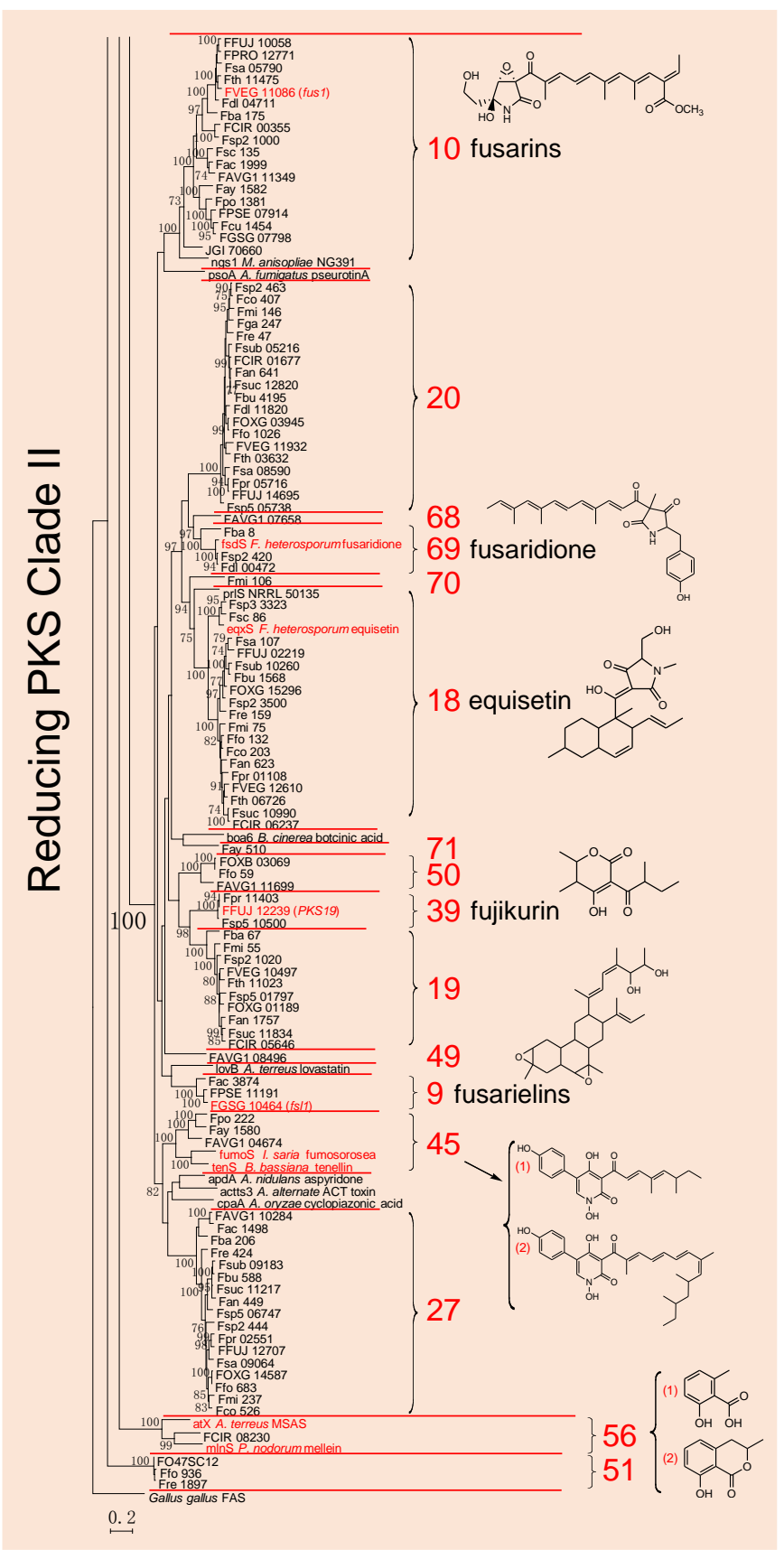


Figure 2.

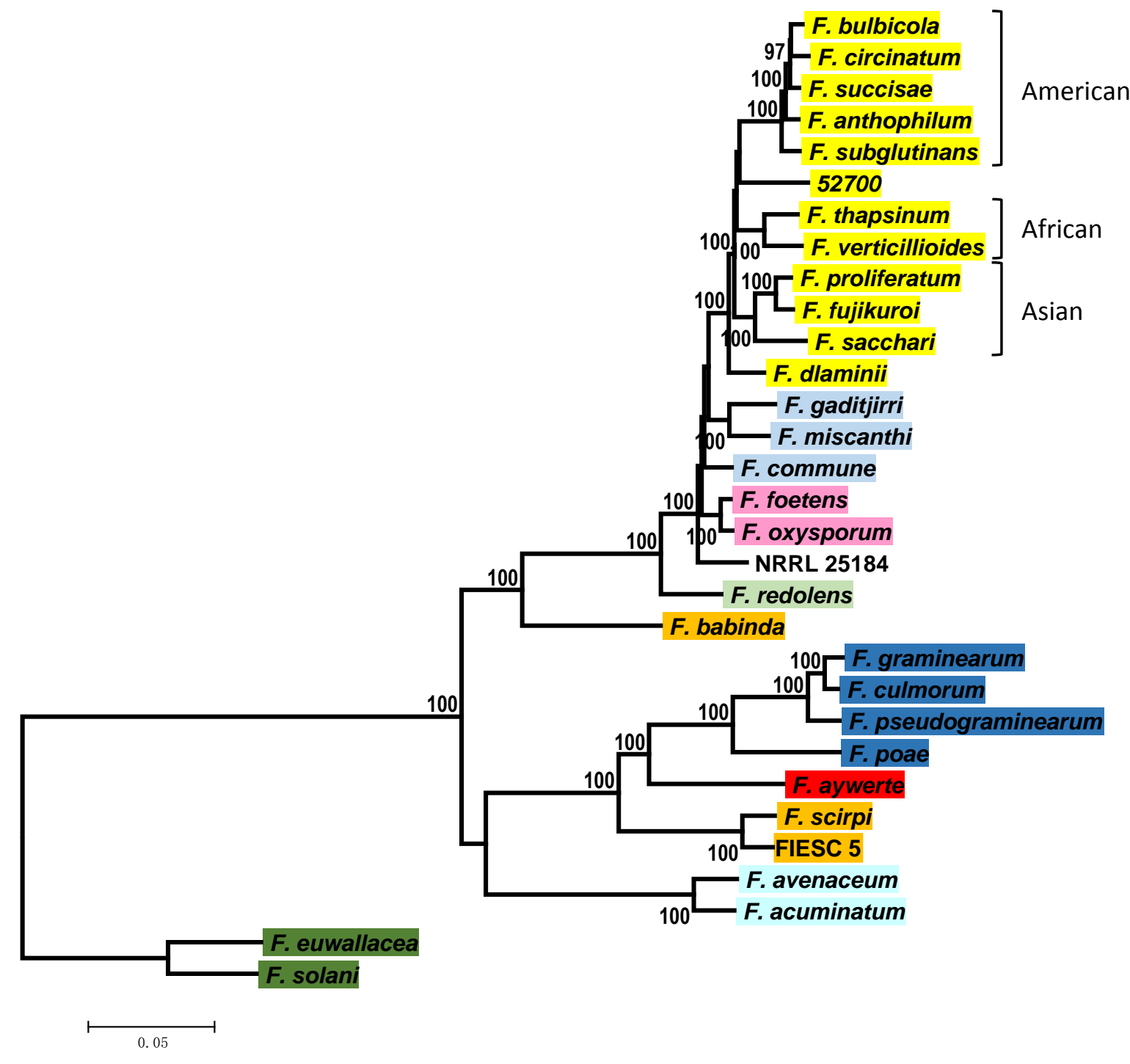

Species Complex Clade 17

Depudecin PKS

fujikuroi

nisikadoi

oxysporum

redolens

babinda

sambucinum

chlamydosporum

incarnatum-equiseti

tricinctum

solani 


\section{Figure 3.}

A.

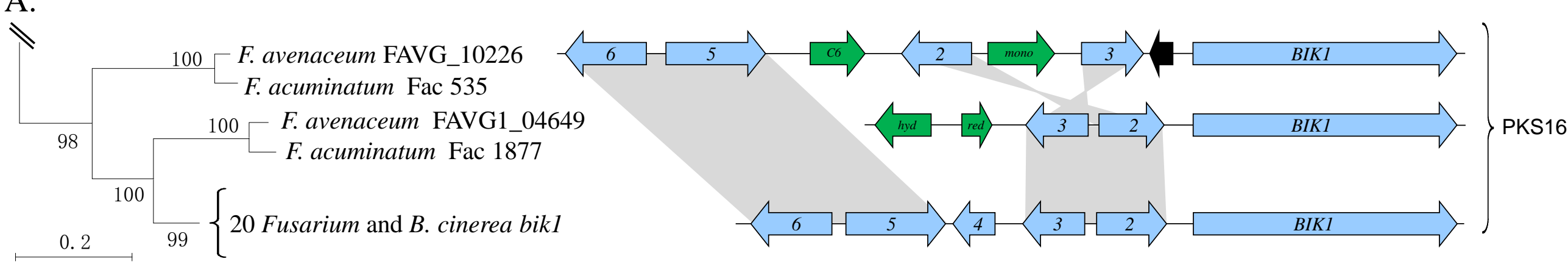

B.

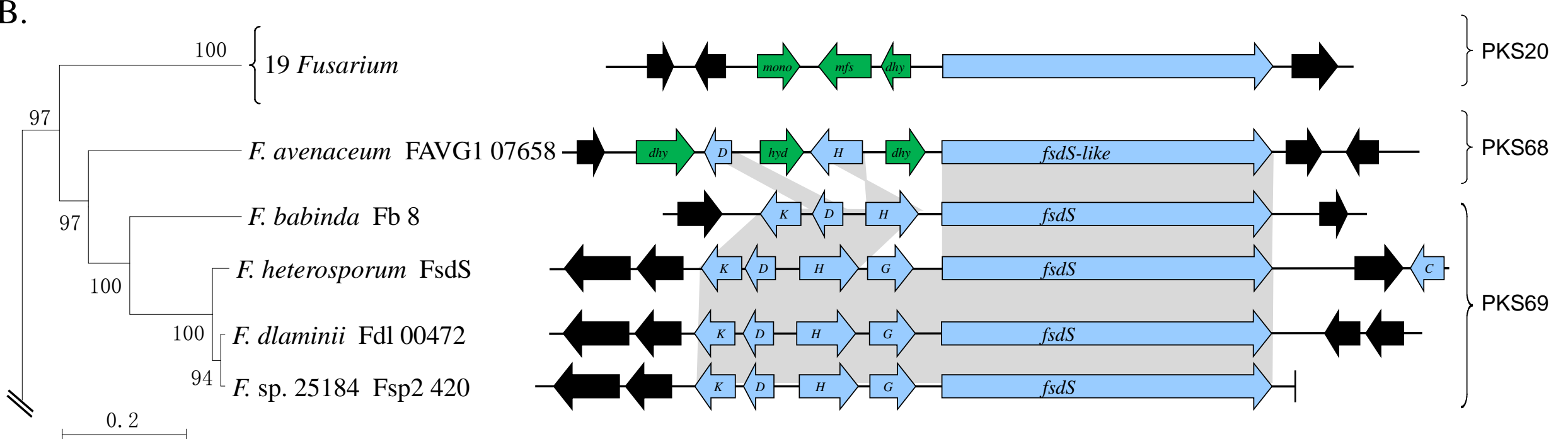


Figure 4. Distribution of PKSs across Fusarium

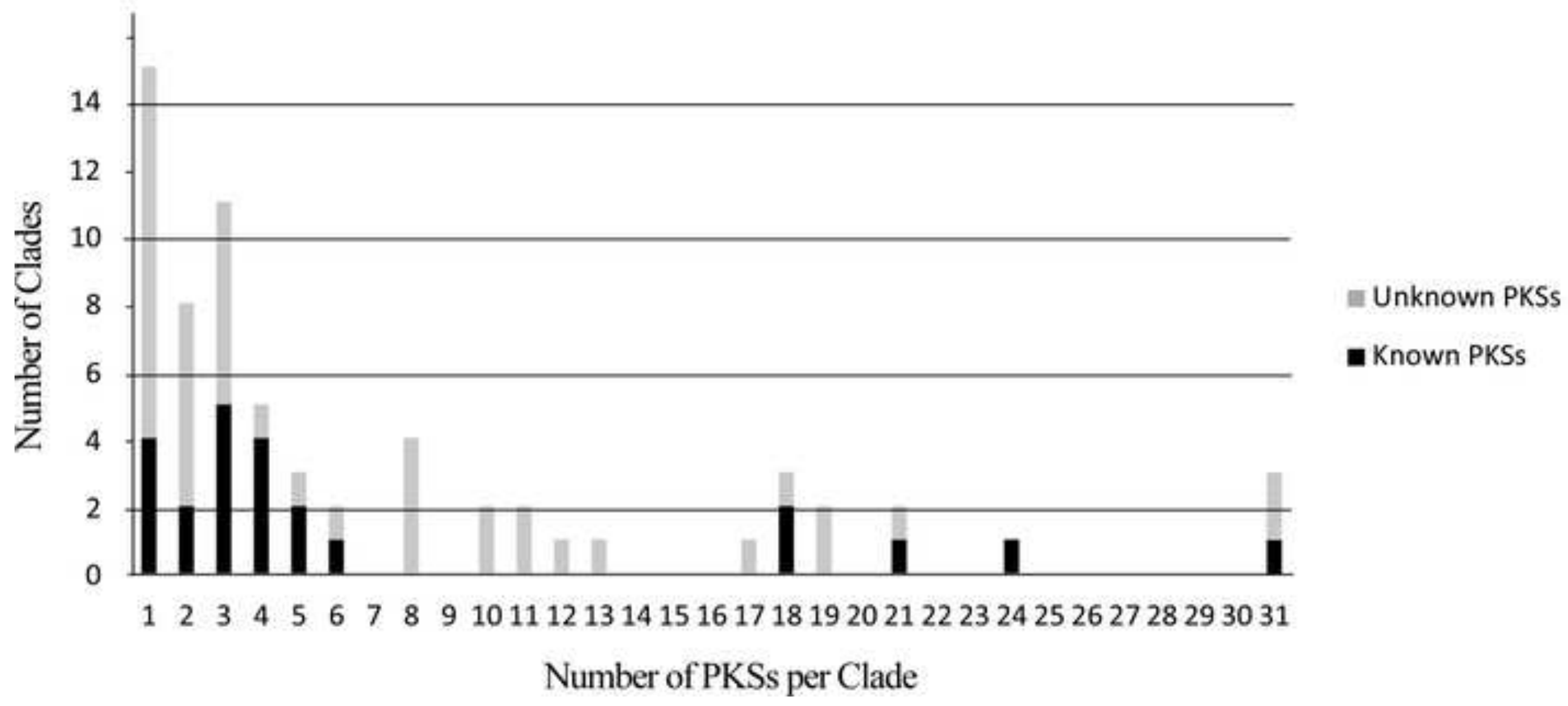




\section{Figure 5.}

\section{A. Alternapyrone cluster homologs: PKS52}

Alternaria solani

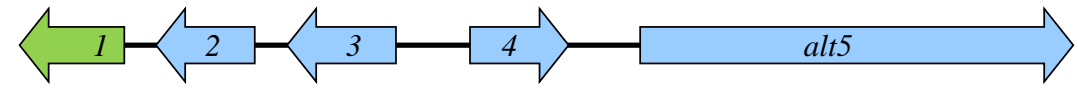

Fusarium dlaminii

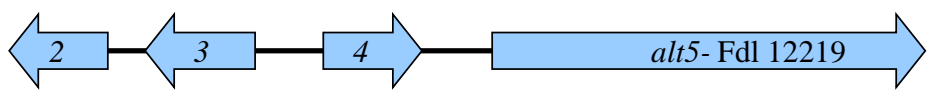

Fusarium thapsinum

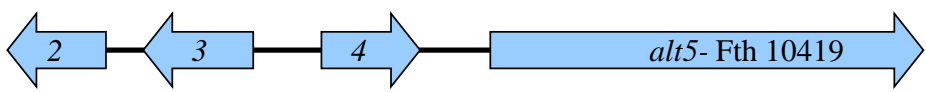

Fusarium anthophilum

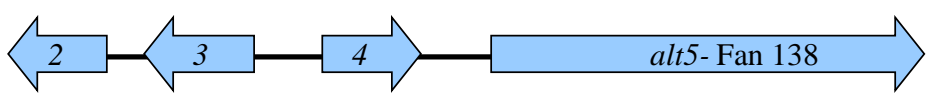

Fusarium sp. 52700

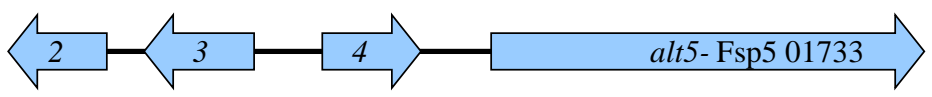

Fusarium sp. 25184

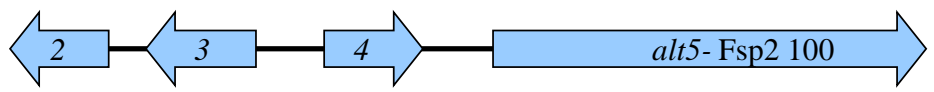

Fusarium graminearum

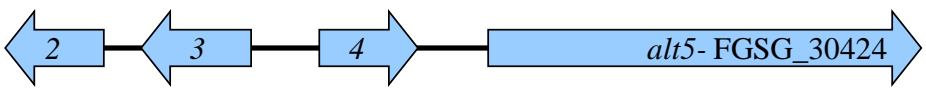

Fusarium bulbicola

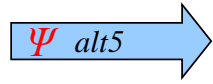

\section{B. Solanapyrone cluster homologs: PKS44}

Alternaria solani

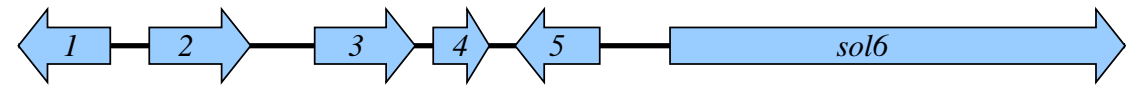

Fusarium avenaceum

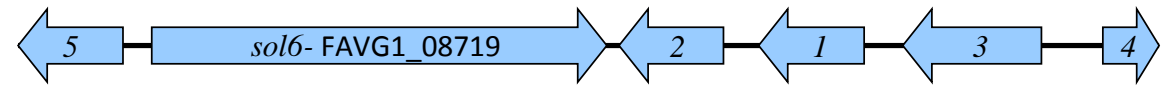

Fusarium acuminatum

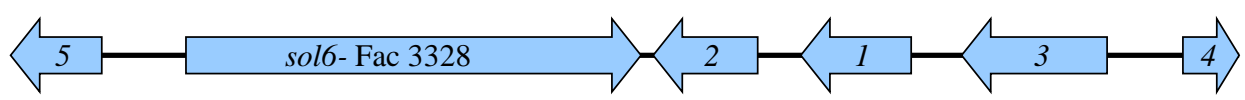

\section{Tenellin cluster homologs: PKS45}

Beauveria bassiana

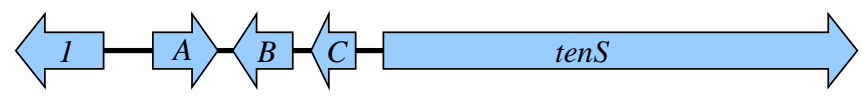

Isaria fumosorosea

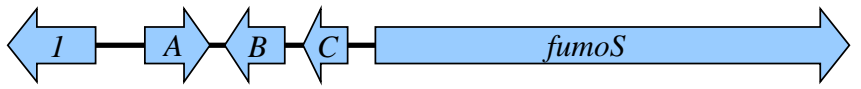

Trichoderma reese $i$

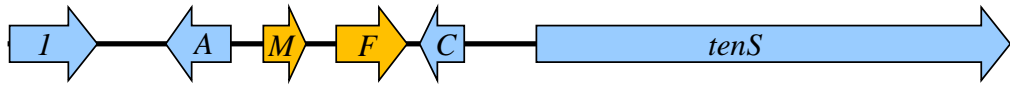

Fusarium avenaceum

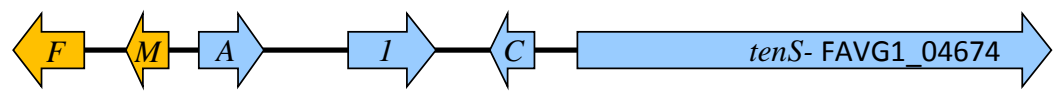

Fusarium aywerte

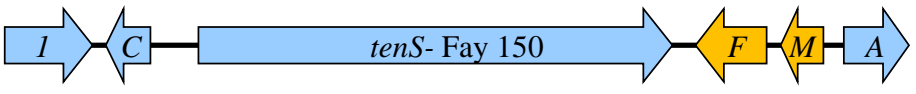

Fusarium poae

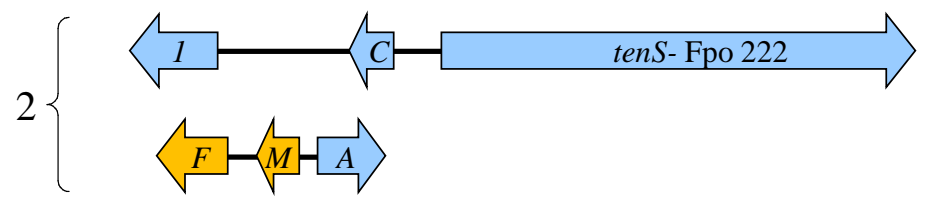


Figure 6. Distribution of Pseudogenes across PKS Clades

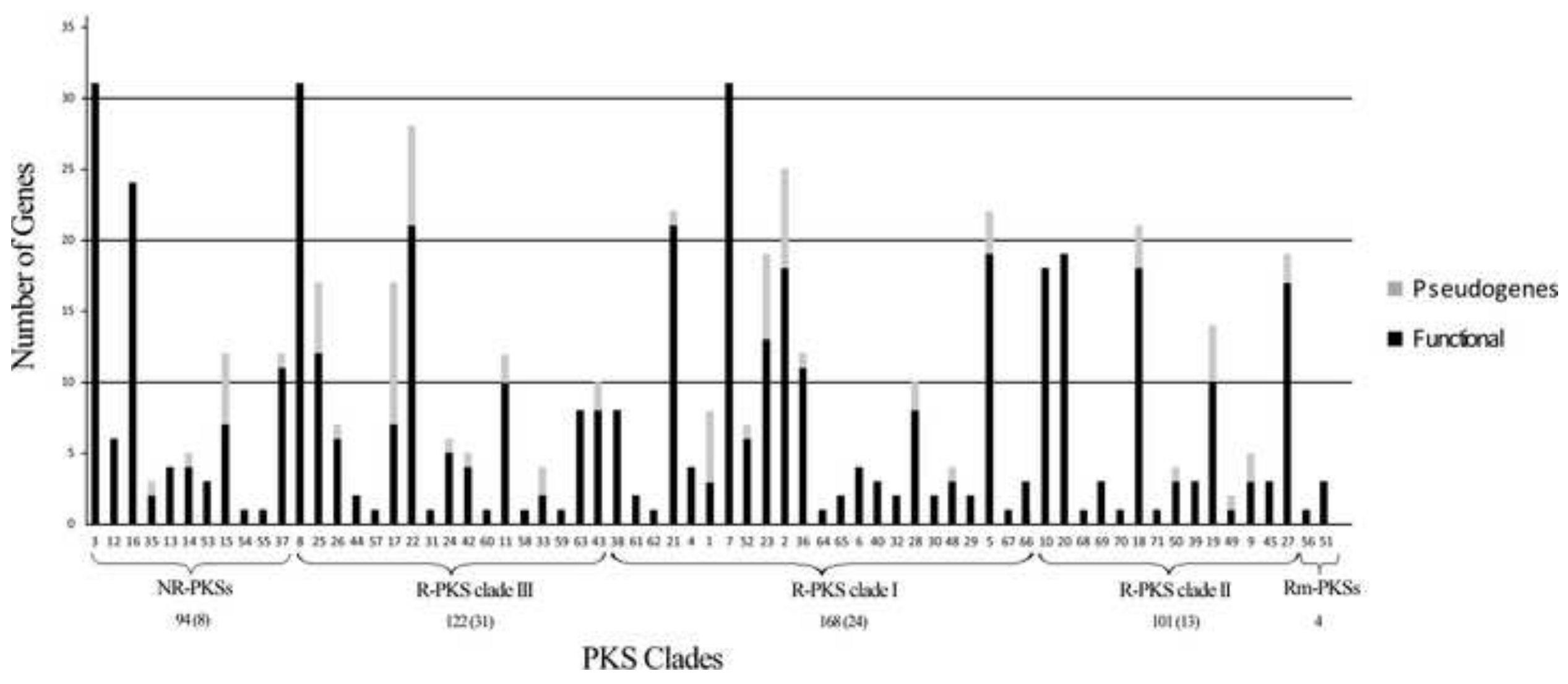

